TRANSACTIONS OF THE

AMERICAN MATHEMATICAL SOCIETY

Volume 363, Number 2, February 2011, Pages 579-609

S 0002-9947(2010)05021-4

Article electronically published on September 21, 2010

\title{
1D SYMMETRY FOR SOLUTIONS OF SEMILINEAR AND QUASILINEAR ELLIPTIC EQUATIONS
}

\author{
ALBERTO FARINA AND ENRICO VALDINOCI
}

\begin{abstract}
Several new 1D results for solutions of possibly singular or degenerate elliptic equations, inspired by a conjecture of De Giorgi, are provided. In particular, 1D symmetry is proven under the assumption that either the profiles at infinity are $2 \mathrm{D}$, or that one level set is a complete graph, or that the solution is minimal or, more generally, $Q$-minimal.
\end{abstract}

\section{Contents}

1. Introduction

1.1. Symmetry from the profiles 5

1.2. Symmetry from level sets being graphs 582

1.3. Symmetry for minimizers 583

1.4. Symmetry from uniform limits 584

1.5. Symmetry for $Q$-minima 584

2. Preliminary results $\quad 585$

2.1. Comparison and calibration results 5

2.2. Barriers 586

2.3. ODE analysis $\quad 587$

2.4. Profile analysis 588

2.5. Proof of Lemma 2.1

2.6. Proof of Lemma 2.2

2.7. Proof of Proposition 2.3

2.8. Proof of Lemma 2.4 590

2.9. Proof of Lemma 2.5 591

2.10. Proof of Corollary 2.6

2.11. Proof of Lemma 2.7 592

2.12. Proof of Corollary 2.8

2.13. Proof of Lemma 2.9 594

2.14. Proof of Proposition 2.10 596

2.15. Proof of Proposition 2.11 597

2.16. Proof of Proposition 2.12

2.17. Proof of Proposition 2.13 600

2.18. Proof of Proposition 2.14 600

Received by the editors April 7, 2008.

2010 Mathematics Subject Classification. Primary 35J92, 35J91, 35J20.

The second author was supported by MIUR Metodi variazionali ed equazioni differenziali nonlineari and FIRB Analysis and Beyond. We thank an anonymous referee whose advice improved the exposition of this paper. 
3. Proof of Theorem 1.10600

4. An alternative proof of Theorem $1.1 \quad 600$

5. Proof of Theorem $1.2 \quad 602$

6. Proof of Theorem $1.3 \quad 604$

7. Proof of Theorem 1.4 604

8. Proof of Theorem $1.5 \quad 605$

9. Proof of Proposition 1.6 606

10. Proof of Theorem $1.7 \quad 606$

11. Proof of Theorem $1.8 \quad 606$

12. Proof of Theorem 1.9

13. Proof of Theorem 1.10 607

References 607

\section{INTRODUCTION}

Given $u: \mathbb{R}^{N} \rightarrow \mathbb{R}$ and $k \in \mathbb{N}$, with $1 \leqslant k \leqslant N$, we say that $u$ possesses $k$ dimensional symmetry (or, for short, that $u$ is $k \mathrm{D}$ ) if there exist $\omega_{1}, \ldots, \omega_{k} \in S^{N-1}$ which are mutually orthogonal and a function $u_{o}: \mathbb{R}^{k} \rightarrow \mathbb{R}$ in such a way that

$$
u(x)=u_{o}\left(\omega_{1} \cdot x, \ldots, \omega_{k} \cdot x\right)
$$

for any $x \in \mathbb{R}^{N}$.

Roughly speaking, $u$ is $k \mathrm{D}$ if it depends only on $k$ variables, namely, the ones in the coordinate directions $\omega_{1}, \ldots, \omega_{k}$.

On page 175 of DG79, the following striking question was posed. Suppose that $u \in C^{2}\left(\mathbb{R}^{N},[-1,1]\right)$ is a solution of

$$
\Delta u+u-u^{3}=0 \text { in the whole } \mathbb{R}^{N},
$$

satisfying

$$
\partial_{x_{N}} u(x)>0 \text { for any } x \in \mathbb{R}^{N} .
$$

Is it true that $u$ is $1 \mathrm{D}$, at least for $N \leqslant 8$ ?

The answer to this question is known to be positive for $N=2,3$, thanks to the results in BCN97, GG98, AC00, AAC01] and, recently, del Pino, Kowalczyk, and Wei dPKW08 have constructed, when $N \geqslant 9$, an example of a solution of (1.1) satisfying (1.2) which is not 1D.

To the best of our knowledge, the question is still open when $4 \leqslant N \leqslant 8$, though a positive answer holds under the additional assumption that

$$
\lim _{x_{N} \rightarrow \pm \infty} u\left(x^{\prime}, x_{N}\right)= \pm 1
$$

for any $x^{\prime} \in \mathbb{R}^{N-1}$, due to Sav03].

The scope of this paper is to give some new 1 D results when $4 \leqslant N \leqslant 8$ under assumptions less restrictive than (1.3).

We will, in fact, deal with a slightly more general form of (1.1), which encompasses possibly singular or degenerate $p$-Laplacian operators $\Delta_{p}$, where, as usual, $p \in(1,+\infty)$ is fixed once and for all and $\Delta_{p} u:=\operatorname{div}\left(|\nabla u|^{p-2} \nabla u\right)$. 
Roughly speaking, we present here essentially three kinds of main results, which will deduce the 1D symmetry of the solution by one of the following hypotheses 1

$(* 1)$ : the $2 \mathrm{D}$ symmetry of limit profiles,

$(* 2)$ : the complete graph of a level set,

$\left({ }^{*} 3\right)$ : the $Q$-minimality of the solution and the sublinearity of level sets.

Here is the formal framework in which we work.

We take $W$ to be a double-well potential. More precisely, we suppose that $W \in$ $C_{\text {loc }}^{1, a}(\mathbb{R}) \cap C_{\text {loc }}^{1,1}((-1,1))$ for some $a \in(0,1)$, that $W(r)>0$ for any $r \in \mathbb{R} \backslash\{-1,+1\}$ and that $W(-1)=W(+1)=0$.

We also suppose that $W^{\prime}(r)=0$ if and only if $r \in\{-1, \kappa, 1\}$, for a suitable $\kappa \in(-1,1)$.

Moreover, we take the following growth conditions near the two wells of $W$. We suppose that there exist some $0<c<1<C$ and some $\theta^{\star} \in(0,1)$ such that:

- For any $\theta \in[0,1]$,

$$
c \theta^{p} \leqslant W(-1+\theta) \leqslant C \theta^{p} \quad \text { and } \quad c \theta^{p} \leqslant W(1-\theta) \leqslant C \theta^{p} .
$$

- For any $\theta \in\left[0, \theta^{*}\right)$,

$$
c \theta^{p-1} \leqslant W^{\prime}(-1+\theta) \leqslant C \theta^{p-1} \text { and } \quad-C \theta^{p-1} \leqslant W^{\prime}(1-\theta) \leqslant-c \theta^{p-1} \text {. }
$$

- $W^{\prime}$ is monotone increasing in $\left(-1,-1+\theta^{\star}\right) \cup\left(1-\theta^{\star}, 1\right)$.

The above assumptions on $W$ are quite common in the literature (see, e.g., VSS06]) and they are satisfied by the standard model $W(r)=\left(1-r^{2}\right)^{p}$.

We will prove 1D symmetry results for

$$
\begin{gathered}
\text { weak solutions } u \in W_{\text {loc }}^{1, p}\left(\mathbb{R}^{N},[-1,1]\right) \text { of } \\
\qquad \Delta_{p} u-W^{\prime}(u)=0 \text { in the whole } \mathbb{R}^{N} .
\end{gathered}
$$

For this, we first observe that (1.2) and standard regularity results imply the existence of $(N-1) \mathrm{D}$ profiles at $\pm \infty$, meaning that there exist $\bar{u}, \underline{u}: \mathbb{R}^{N-1} \rightarrow \mathbb{R}$ in such a way that

$$
\lim _{x_{N} \rightarrow+\infty} u\left(x^{\prime}, x_{N}\right)=\bar{u}\left(x^{\prime}\right) \text { and } \lim _{x_{N} \rightarrow-\infty} u\left(x^{\prime}, x_{N}\right)=\underline{u}\left(x^{\prime}\right)
$$

for any $x^{\prime} \in \mathbb{R}^{N-1}$.

\footnotetext{
${ }^{1}$ Typical examples of cases $\left({ }^{*} 1\right),\left(*^{2}\right)$ and $\left({ }^{*} 3\right)$ will be Theorems 1.11 .3 and 1.10 respectively.

Though the proof of our results will be quite technical, the expert reader may find it useful to get an idea of the methods used from the beginning.

In case $(* 1)$, one of the main steps is to prove that the limit profiles (that we will formally introduce in (1.5) below) are 1D. This will be accomplished by using inequality (4.17) in [FSV08, then by showing that these profiles are global minimizers and so is $u$.

Accordingly, if one profile is not constant, then a rescaling argument (which shows that a typical level set of the solution converges to a minimal surface) implies that the solution is 1D, thanks to Corollary 7 of [FV08]. Finally, this gives that the two profiles are constant and equal to \pm 1 and so the solution itself is $1 \mathrm{D}$ up to dimension $N \leqslant 8$, because of the results in VSS06.

In case $(* 2)$, the main idea is that if one level set of the solution is a complete graph, then one of the limit profiles is constant, due to some results in [Far99, Far03]. Then, an energy estimate shows that the other profile is also constant, and this reduces the situation to the one treated in case $\left({ }^{*} 1\right)$, which implies the $1 \mathrm{D}$ symmetry result.

As for case $\left({ }^{*} 3\right)$, minimal solutions are well-understood (up to dimension 7 , or 8 when they are monotone); see [VSS06]. Here, we extend the symmetry result to $Q$-minimizers which are either low-dimensional or with sublinear level sets. To do this, we will rescale the solution and again use Corollary 7 of [FV08.
} 
In fact, well-known regularity results give that the above limits hold in $C_{\text {loc }}^{1, \alpha}$ (see [DiB83, Tol84]). Condition (1.3) requires $\bar{u}$ and $\underline{u}$ to be simply \pm 1 . Theorem 1.1 below will show that such a condition can be weakened and still 1D symmetry holds.

We recall that particularly important solutions of our PDE are the minimizers, namely the ones that satisfy

$$
\int_{B_{R}^{N}} \frac{1}{p}|\nabla(u+\eta)|^{p}+W(u+\eta) d x \geqslant \int_{B_{R}^{N}} \frac{1}{p}|\nabla u|^{p}+W(u) d x
$$

for any $\eta \in C_{0}^{\infty}\left(B_{R}^{N}\right)$ and any $R>0$.

As usual, $B_{R}^{N}$ is used to denote the open $N$-dimensional ball centered at the origin with radius $R$. In jargon, condition (1.6) is stated by saying that $u$ is a global minimizer, following JM04], or a class A minimizer, according to the nomenclature of [VSS06].

Of course, if $u \in W_{\text {loc }}^{1, p}\left(\mathbb{R}^{N},[-1,1]\right)$ satisfies (1.6), then it satisfies (1.4).

1.1. Symmetry from the profiles. We now present some results obtaining the $1 \mathrm{D}$ symmetry from symmetry assumptions on the profiles $\bar{u}$ and $\underline{u}$ :

Theorem 1.1 (Symmetry if both the profiles are 2D). Let $u$ be as in (1.2) and (1.4).

Let $\bar{u}$ and $\underline{u}$ be as in (1.5).

Suppose that both $\bar{u}$ and $\underline{u}$ are $2 D$.

Then, $\bar{u}$ is identically $+1, \underline{u}$ is identically -1 and $u$ satisfies (1.6).

Also, if $N \leqslant 8$, then $u$ is $1 D$.

In dimension $N \leqslant 4$, under the additional assumptions that $W \in C^{2}(\mathbb{R})$ and $p=2$ (as in the classical case of [DG79]), the claim of Theorem 1.1 also holds true when only one profile is $2 \mathrm{D}$, according to the following result:

Theorem 1.2 (Symmetry if one profile is 2D). Let $u$ be as in (1.2) and (1.4).

Let $\bar{u}$ and $\underline{u}$ be as in (1.5).

Suppose that $2 \leqslant N \leqslant 4$, that $p=2$ and that $W \in C^{2}(\mathbb{R})$.

Assume that either $\bar{u}$ or $\underline{u}$ is $2 D$.

Then, $u$ is $1 D$ and it satisfies (1.6).

1.2. Symmetry from level sets being graphs. The next result proves $1 \mathrm{D}$ symmetry under the assumption that one level set, say $\{u=c\}$, is a complete graph over the entire $\mathbb{R}^{N-1}$, meaning that there exists $\Gamma: \mathbb{R}^{N-1} \rightarrow \mathbb{R}$ in such a way that

$$
\{u=c\}=\left\{\left(x^{\prime}, x_{N}\right) \in \mathbb{R}^{N-1} \times \mathbb{R} \text { s.t. } x_{N}=\Gamma\left(x^{\prime}\right)\right\} .
$$

We remark that condition (1.7) is, of course, compatible with (1.2), but it is not implied by it (a counterexample being $u\left(x^{\prime}, x_{N}\right)=\gamma\left(x_{1}+e^{x_{N}}\right)$, for a monotone and bounded function $\gamma$ ).

Theorem 1.3 (Symmetry if one level set is a complete graph in the semilinear case). Suppose that $p=2$ and $W(r)=\left(1-r^{2}\right)^{2}$. Let $u$ be as in (1.2) and (1.4).

Let $\bar{u}$ and $\underline{u}$ be as in (1.5) and suppose that (1.7) holds.

Then, $\bar{u}$ is identically $+1, \underline{u}$ is identically -1 and $u$ satisfies (1.6).

Moreover, if $2 \leqslant N \leqslant 8$, then $u$ is $1 D$. 
Though 2 we do not have a complete extension of Theorem 1.3 to the quasilinear case and to more wild double-well potentials, we can prove the results contained in the subsequent Theorems 1.4 and 1.5 .

Theorem 1.4 (Symmetry if one level set is a complete graph in the quasilinear case). Suppose that either

$$
N \leqslant 4
$$

or

$$
p=2 \text { and } N \leqslant 5 \text {. }
$$

Let $u$ be as in (1.2) and (1.4). Assume that (1.7) holds.

Then, $\bar{u}$ is identically $+1, \underline{u}$ is identically $-1, u$ is $1 D$ and it satisfies (1.6).

Theorem 1.5 (Symmetry if the $\kappa$-level set is a complete graph). Suppose that $p \geqslant N-3$ and let $u$ be as in (1.2) and (1.4).

Suppose that $\{u=\kappa\}$ is a complete graph over the entire $\mathbb{R}^{N-1}$.

Then, the theses of Theorem 1.3 hold true.

We note that Theorem 1.5 requires both a bound on $p$ with respect to the dimension $N$ and that the level set corresponding to the maximum of $W$ is a complete graph, while Theorem 1.4 under assumption (1.8) works when any level set is a graph and for any $p>1$, but it requires, for $p \neq 2$, a stronger assumption on the dimension.

1.3. Symmetry for minimizers. The next result deals with the profiles of the minimizing solutions:

Proposition 1.6. Let $u \in W_{\mathrm{loc}}^{1, p}\left(\mathbb{R}^{N},[-1,1]\right)$ be as in (1.2) and (1.6).

Let $\bar{u}$ be as in (1.5) and suppose that

$$
\bar{u} \text { is } 7 D \text {. }
$$

Then, $\bar{u}$ is constantly equal to +1 .

Analogously, if $\underline{u}$ is $7 D$, then it is constantly equal to -1 .

Of course, if $N \leqslant 8$, then condition (1.10) is automatically satisfied. In this spirit, we now present a result for the case of minimizers in dimension 8:

Theorem 1.7 (Symmetry for monotone minimizers). Let $N=8$. Let $u \in$ $W_{\mathrm{loc}}^{1, p}\left(\mathbb{R}^{N},[-1,1]\right)$ be as in (1.2), satisfying (1.6). Then, $u$ is $1 D$.

Theorem 1.7 is a non-trivial generalization of Theorem 1.4 of VSS06]. More precisely, Theorem 1.4 of VSS06] proved the claim in Theorem 1.7 here under the additional assumption (1.3) (such an additional assumption was crucial in VSS06] to obtain a graph property for level sets; see page 80 there).

\footnotetext{
${ }^{2}$ In the statement of Theorem 1.3 the assumption that $W$ is exactly the standard doublewell potential can be weakened. Indeed, following [Far03, the structural assumption needed for Theorem 1.3 is that there exist $\mu, \delta>0$ such that

$$
\frac{W^{\prime}(r)}{r-\kappa} \leqslant-\mu
$$

for any $r \in(\kappa-\delta, \kappa+\delta) \backslash\{\kappa\}$.
} 
1.4. Symmetry from uniform limits. The next result deals with the uniform limit case for minimal solutions (no monotonicity assumption is needed); we will point out in such results that the control of only one limit is enough to obtain the $1 \mathrm{D}$ symmetry.

Theorem 1.8 (Symmetry if one limit is uniform). Let $u \in W_{\text {loc }}^{1, p}\left(\mathbb{R}^{N},[-1,1]\right)$ satisfy (1.6). Suppose that either

$$
\lim _{x_{N} \rightarrow+\infty} u\left(x^{\prime}, x_{N}\right)=1 \quad \text { uniformly for } x^{\prime} \in \mathbb{R}^{N-1}
$$

or

$$
\lim _{x_{N} \rightarrow-\infty} u\left(x^{\prime}, x_{N}\right)=-1 \quad \text { uniformly for } x^{\prime} \in \mathbb{R}^{N-1} .
$$

Then, $u$ is $1 D$.

We recall that, for $p=2$, any solution of (1.1) satisfying both (1.11) and (1.12) also satisfies (1.2) (see, for instance, GG98, Far99, Far01) and so (1.6) (see, e.g., AC00, AAC01 and Lemma 9.1 in VSS06]). Therefore, Theorem 1.8 contains, as a particular case, the fact that, for $p=2$, solutions of (1.1) with uniform limits \pm 1 are 1D in any dimension $N$. This statement, known in the literature under the name of Gibbons conjecture, was first proven independently and with different methods by Far99, BBG00, BHM00.

1.5. Symmetry for $Q$-minima. We now deal with $Q$-minima, with the intention of carrying out the research started in [FV08]. For this, we recall that, given $Q \geqslant 1$, $u$ is said to be a $Q$-minimum in the bounded domain $\Omega \subset \mathbb{R}^{N}$ if

$$
Q \int_{\Omega} \frac{1}{p}|\nabla(u+\eta)|^{p}+W(u+\eta) d x \geqslant \int_{\Omega} \frac{1}{p}|\nabla u|^{p}+W(u) d x
$$

for any $\eta \in C_{0}^{\infty}(\Omega)$.

Of course, for $Q=1$, (1.13) boils down to (1.6).

Theorem 1.9 (Symmetry for $Q$-minimal monotone solutions). Let $2 \leqslant N \leqslant 4$. Let $u$ be as in (1.2) and (1.4). Suppose that (1.13) holds. Then, $u$ is $1 D$.

For minimal solutions or, more generally, for $Q$-minimal solutions with $Q$ close to 1 , it is possible to obtain the 1D symmetry in any dimension, provided that one level set grows less than linearly, according to the subsequent Theorem 1.10.

For this, given $\xi \in S^{N-1}$, we denote by $\pi_{\xi}$ the projection along $\xi^{\perp}:=\{v \in$ $\mathbb{R}^{N}$ s.t. $\left.v \cdot \xi=0\right\}$, that is,

for any $w \in \mathbb{R}^{N}$.

$$
\pi_{\xi} w:=w-(w \cdot \xi) \xi
$$

With the above notation, we have the following result:

Theorem 1.10 (Symmetry for $Q$-minimal solutions with $Q$ close to 1). Let $u$ be as in (1.4), with $W(r)=\left(1-r^{2}\right)^{p}$. Suppose that (1.13) holds and that there exists $\theta \in(-1,1), \xi \in S^{N-1}$ and $\Phi: \mathbb{R}^{N} \rightarrow[0,+\infty)$ in such a way that

$$
\{u=\theta\} \subseteq\left\{|x \cdot \xi| \leqslant \Phi\left(\pi_{\xi} x\right)\right\}
$$

and, for any $K>0$,

$$
\lim _{\epsilon \rightarrow 0}\left(\epsilon \sup _{w \in \xi^{\perp},|w| \leqslant K} \Phi(w / \epsilon)\right)=0 .
$$


Then, there exists a suitable constant $\kappa_{o}>0$ such that if $Q \leqslant 1+\kappa_{o}$, we have that $u$ is $1 D$.

The proofs of our results will rely on a profile analysis and they will combine several result:3 of [Far03, VSS06, FV08, FSV08].

We remark that the results of this paper are, to the best of our knowledge, new even in the semilinear case $p=2$.

This paper is organized as follows. We present some preliminary results, some of them interesting in themselves, in $\S \$ 2.1$ 2.4. These auxiliary results are proven in $\S \S 2.5,2.18$. Two proofs of Theorem 1.1 are given in $\S 3$ and $\S 4$ the first one uses some calibration results; the second one is calibration independent. The other main results are proven in $\S \$ 513$.

\section{Preliminary Results}

2.1. Comparison and calibration results. The proof of our main results will make use of some preliminary considerations. We list here such auxiliary tools, postponing the proofs for the reader's convenience.

First, we consider a strong comparison principle in the form needed for this paper:

Lemma 2.1. Let $\Omega \subseteq \mathbb{R}^{N}$ be an open connected set. For $i=1,2$, let $u_{i} \in$ $C^{1}(\Omega,[-1,1])$ be weak solutions of $\Delta_{p} u_{i}=W^{\prime}\left(u_{i}\right)$ in $\Omega$, with $u_{1}(x) \leqslant u_{2}(x)$ for any $x \in \Omega$.

Assume that one of the following conditions holds:

(i): $p=2$,

(ii): $\left\{\left|\nabla u_{1}\right|+\left|\nabla u_{2}\right|=0\right\}=\emptyset$,

(iii): $u_{1}$ is identically -1 ,

(iv): $u_{2}$ is identically +1 .

Then, either $u_{1}(x)<u_{2}(x)$ for any $x \in \Omega$ or $u_{1}$ is identically equal to $u_{2}$.

We then point out some preliminary considerations on how the $Q$-minimality of a solution reflects into the same property for the profiles. These observations will lead to the subsequent Proposition 2.3, which is interesting in itself, being a generalization of Theorem 1.3 of [JM04, where an analogous result is obtained in the case $p=2$ and $Q=1$.

We first recall the calibration result asserting that monotone solutions are minimizers for perturbations staying between $\underline{u}$ and $\bar{u}$ :

Lemma 2.2. Suppose that $u$ is as in (1.2) and (1.4).

Then, $u$ satisfies (1.6) for any $\eta \in C_{0}^{\infty}\left(B_{R}^{N}\right)$, for any $R>0$, provided that

$$
\underline{u}\left(x^{\prime}\right) \leqslant u(x)+\eta(x) \leqslant \bar{u}\left(x^{\prime}\right)
$$

for any $x=\left(x^{\prime}, x_{n}\right) \in \mathbb{R}^{N}$.

\footnotetext{
${ }^{3}$ We observe that, in our general setting, $W^{\prime}$ is not locally Lipschitz when $p<2$; thus the results of [FSV08] are not, in principle, directly applicable. However, we will obtain from the strong comparison principle of Lemma 2.1 that $|u|<1$. Then, since $W^{\prime}$ is locally Lipschitz inside $(-1,1)$, we will be in a position of using the results of [FSV08].

Also, we point out that the ODE analysis of [FSV08] is valid for continuous nonlinearities $f:=$ $-W^{\prime}$.
} 
Next is the generalization of Theorem 1.3 of [JM04, which fits our scopes:

Proposition 2.3. Suppose that $u$ is as in (1.2) and (1.4).

Suppose also that there exists $Q \geqslant 1$ such that

$$
\begin{aligned}
Q \int_{\Omega^{\prime}} \frac{1}{p}|\nabla(\underline{u}+\eta)|^{p}+W(\underline{u}+\eta) d x^{\prime} & \geqslant \int_{\Omega^{\prime}} \frac{1}{p}|\nabla \underline{u}|^{p}+W(\underline{u}) d x^{\prime} \\
\text { and } Q \int_{\Omega^{\prime}} \frac{1}{p}|\nabla(\bar{u}+\eta)|^{p}+W(\bar{u}+\eta) d x^{\prime} & \geqslant \int_{\Omega^{\prime}} \frac{1}{p}|\nabla \bar{u}|^{p}+W(\bar{u}) d x^{\prime}
\end{aligned}
$$

for any bounded domain $\Omega^{\prime} \subset \mathbb{R}^{N-1}$ and any $\eta \in C_{0}^{\infty}\left(\Omega^{\prime}\right)$.

Then, $u$ satisfies (1.13) for any bounded domain $\Omega \subset \mathbb{R}^{N}$ and any $\eta \in C_{0}^{\infty}(\Omega)$.

2.2. Barriers. The proofs of Theorems 1.1 and 1.3 will also rely on the following auxiliary results. The first deals with a barrier. The second is a flatness result. These results are first stated and proved later on, in order not to interrupt the thread of the argument.

Lemma 2.4. Let $a \in(0,1), \lambda \in(-1,1)$ and $\mu \in(-1, \lambda)$. Let $\tilde{W} \in C_{\mathrm{loc}}^{1, a}(\mathbb{R}) \cap$ $C_{\text {loc }}^{1,1}((-1,1))$ be such that

$$
\begin{gathered}
\tilde{W}(r) \geqslant \tilde{W}(-1) \text { for any } r \leqslant \mu, \\
\tilde{W}(r)=W(r) \text { for any } r \geqslant \lambda
\end{gathered}
$$

and

$$
\inf _{(-\infty, \lambda]} \tilde{W}>0
$$

Given any $R \geqslant 1$, there exists $\beta_{R} \in C^{1}\left(B_{R}^{N},(-1,1)\right)$, such that $\beta_{R}=-1$ on $\partial B_{R}^{N}$,

$$
\Delta_{p} \beta_{R}-\tilde{W}^{\prime}\left(\beta_{R}\right)=0
$$

in $B_{R}^{N}$ and

$$
\lim _{R \rightarrow+\infty}\left(\sup _{B_{R}^{N}} \beta_{R}\right)=+1
$$

Lemma 2.5. Let $v \in W_{\text {loc }}^{1, p}\left(\mathbb{R}^{N},[-1,1]\right)$ be a weak solution of (1.4) such that

$$
\sup _{\mathbb{R}^{N}} v=1
$$

Assume that either $p=2$ or $\{\nabla v=0\}=\emptyset$.

Then, either $\inf _{\mathbb{R}^{N}} v=-1$ or $v(x)=1$ for any $x \in \mathbb{R}^{N}$.

Given $R>0$ and $v \in W^{1, p}\left(B_{R}^{N}\right) \cap L^{\infty}\left(B_{R}^{N}\right)$, we define

$$
E_{R}(v):=\int_{B_{R}^{N}} \frac{1}{p}|\nabla v(x)|^{p}+W(v(x)) d x .
$$


A consequence of Lemma 2.5 is the following.

Corollary 2.6. Suppose that $v \in W_{\mathrm{loc}}^{1, p}\left(\mathbb{R}^{N},[-1,1]\right)$ is a weak solution of (1.4) so that

$$
\inf _{\mathbb{R}^{N}} v>-1
$$

and

$$
\liminf _{R \rightarrow+\infty} \frac{E_{R}(v)}{R^{N}}=0
$$

Assume that either $p=2$ or $\{\nabla v=0\}=\emptyset$.

Then, $v(x)=1$ for any $x \in \mathbb{R}^{N}$.

2.3. ODE analysis. We classify solutions of the associated ODE, as follows:

Lemma 2.7. Let $h \in W_{\text {loc }}^{1, p}(\mathbb{R},[-1,1])$ be a weak solution of

$$
\left(\left|h^{\prime}\right|^{p-2} h^{\prime}\right)^{\prime}-W^{\prime}(h)=0
$$

in the whole $\mathbb{R}$.

Then, $h$ must satisfy one of the following possibilities:

(P1): $h$ is constantly equal to either $-1, \kappa$ or +1 ,

(P2): $\left\{h^{\prime}=0\right\}=\emptyset$, and $h$ attains at infinity limits -1 (on one side) and +1 (on the other side),

(P3): $h^{\prime}(t) \neq 0$ for any $t$ in a bounded interval of the form $\left(\beta_{1}, \beta_{2}\right)$ with

$$
\begin{aligned}
& h^{\prime}\left(\beta_{1}\right)=h^{\prime}\left(\beta_{2}\right)=0, \\
& W\left(h\left(\beta_{1}\right)\right)=W\left(h\left(\beta_{2}\right)\right)=W\left(\inf _{\mathbb{R}} h\right)=W\left(\sup _{\mathbb{R}} h\right), \\
& h\left(\beta_{1}\right), h\left(\beta_{2}\right) \in(-1,1), \\
& \left\{h\left(\beta_{1}\right), h\left(\beta_{2}\right)\right\}=\left\{\inf _{\mathbb{R}} h, \sup _{\mathbb{R}} h\right\}=\left\{\min _{\mathbb{R}} h, \max _{\mathbb{R}} h\right\} .
\end{aligned}
$$

We remark that Lemma 2.7 heavily depends on the growth and shape assumptions we took on $W$. For a more general potential, the result is actually false and, for instance, plateaus may be developed (see, e.g., Propositions 7.2 and 7.3 in [FSV08).

The result in Lemma 2.7 may also be strengthened as follows:

Corollary 2.8. Let the setting of Lemma 2.7 hold. Then, the following conditions are equivalent:

(S1): either (P2) or (P3) holds,

(S2): there exists $t_{-}, t_{+} \in \mathbb{R}$ for which $h\left(t_{-}\right)<\kappa<h\left(t_{+}\right)$.

We now classify the minimal solutions of the associated ODE:

Lemma 2.9. Let $h \in W_{\text {loc }}^{1, p}(\mathbb{R},[-1,1])$ be a weak solution of (2.11) in the whole $\mathbb{R}$. Then, the following conditions are equivalent:

(a): $h$ is either constantly equal to -1 or +1 , or $\left\{h^{\prime}=0\right\}=\emptyset$ with limits +1 and -1 at infinity,

(b): h satisfies (1.6). 
2.4. Profile analysis. We present some geometric properties and minimality features for $1 \mathrm{D}$ profiles. For this, we take $u$ as in (1.2) and (1.4), and $\bar{u}, \underline{u}$ as in (1.5).

Proposition 2.10. Suppose that both $\underline{u}$ and $\bar{u}$ are $1 D$.

Then:

(C1): either $\underline{u}$ is identically equal to -1 or it does not have any critical points and it converges to -1 and +1 at infinity, and

(C2): either $\bar{u}$ is identically equal to +1 or it does not have any critical points and it converges to -1 and +1 at infinity.

Also, $\underline{u}$ and $\bar{u}$ satisfy (1.6) and

$$
\int_{B_{R}^{N}} \frac{1}{p}|\nabla \underline{u}|^{p}+W(\underline{u}) d x+\int_{B_{R}^{N}} \frac{1}{p}|\nabla \bar{u}|^{p}+W(\bar{u}) d x \leqslant \bar{C} R^{N-1}
$$

for a suitable $\bar{C}>0$, for any $R>0$.

We remark that, in our general setting, a statement such as the one in Proposition 2.10 does not easily follow from standard arguments. Indeed, since $W$ is not assumed to be in $C^{2}(\mathbb{R})$, the linearized equation may not behave continuously at infinity. Moreover, since we allow the possibility of $W^{\prime \prime}(\kappa)$ to vanish, the function constantly equal to $\kappa$ may be a stable profile which could not be excluded by the stability methods in $\mathrm{AC00}$, AAC01].

A byproduct of our analysis is that energy bounds imply 1D symmetries of the profiles:

Proposition 2.11. Suppose that

$$
\int_{B_{R}^{N-1}}\left|\nabla \underline{u}\left(x^{\prime}\right)\right|^{p} d x^{\prime} \leqslant C R^{2}
$$

for some $C>0$.

Then, $\underline{u}$ is $1 D$.

Also, if $N \leqslant 4$ and

$$
\int_{B_{R}^{N-1}} \frac{1}{p}\left|\nabla \underline{u}\left(x^{\prime}\right)\right|^{p}+W(\underline{u}) d x^{\prime} \leqslant C R^{2}
$$

for some $C>0$, then, both $\bar{u}$ and $\underline{u}$ are $1 D$.

Analogous results hold by exchanging the roles of $\bar{u}$ and $\underline{u}$.

Here is another criterion for 1D symmetry of profiles:

Proposition 2.12. If $\underline{u}$ is $2 D$, then it is $1 D$. Analogously, if $\bar{u}$ is $2 D$, then it is $1 D$.

Below we rule out strictly monotone profiles for minimal solutions:

Proposition 2.13. Let $u$ satisfy (1.2) and (1.6).

If $\bar{u}$ is $1 D$, then it is constantly equal to +1 .

Analogously, if $\underline{u}$ is $1 D$, then it is constantly equal to -1 .

A similar result can be proven without the minimality assumption, when both the profiles are $1 \mathrm{D}$, according to the following result. 
Proposition 2.14. Let $u \in W_{\text {loc }}^{1, p}\left(\mathbb{R}^{N},[-1,1]\right)$ be as in (1.2) and (1.4).

Let $\bar{u}$ and $\underline{u}$ be as in (1.5) and suppose that they are both $1 D$.

Then, $\bar{u}$ is constantly equal to +1 and $\underline{u}$ is constantly equal to -1 .

Note that in Proposition 2.13 where minimality is assumed, it is possible to control independently any profile. On the contrary, in Proposition 2.14, minimality is not assumed, but then we need to suppose that both the profiles are 1D to fully classify them. Lemma 2.9 and Proposition 2.10 also reflect a similar feature.

2.5. Proof of Lemma 2.1. Case (i) is classical.

If either (iii) or (iv) holds, the claim of Lemma 2.1 follows from Váz84.

If (ii) holds, we first use (iii) and (iv) to deduce that $|u(x)|<1$ for any $x \in \Omega$. Then, the claim of Lemma 2.1 is a consequence of Dam98.

For related results and further comments, the interested reader may also look at [PSZ99, SV05].

2.6. Proof of Lemma 2.2. See Theorem 4.5 in AAC01, as exploited, for instance, in Theorem 10.4 of [DG02].

2.7. Proof of Proposition 2.3. Given any bounded domain $U \subset \mathbb{R}^{N}$ and any $\phi \in C_{0}^{\infty}(U)$, we write $\phi_{x_{N}}\left(x^{\prime}\right):=\phi\left(x^{\prime}, x_{N}\right)$ for any fixed $x_{N} \in \mathbb{R}$. We also denote $U_{x_{N}}:=\left\{x^{\prime} \in \mathbb{R}^{N-1}\right.$ s.t. $\left.\left(x^{\prime}, x_{N}\right) \in U\right\}$. Note that $U_{x_{N}}$ is bounded, since so is $U$, and that $\phi_{x_{N}} \in C_{0}^{\infty}\left(U_{x_{N}}\right)$.

Therefore, we deduce from (2.1) that

$$
\begin{aligned}
& Q \int_{U} \frac{1}{p}|\nabla(\underline{u}+\phi)|^{p}+W(\underline{u}+\phi) d x \\
= & Q \int_{\mathbb{R}} \int_{U_{x_{N}}} \frac{1}{p}\left|\nabla\left(\underline{u}\left(x^{\prime}\right)+\phi_{x_{N}}\left(x^{\prime}\right)\right)\right|^{p}+W\left(\underline{u}\left(x^{\prime}\right)+\phi_{x_{N}}\left(x^{\prime}\right)\right) d x^{\prime} d x_{N} \\
\geqslant & \int_{\mathbb{R}} \int_{U_{x_{N}}} \frac{1}{p}\left|\nabla\left(\underline{u}\left(x^{\prime}\right)\right)\right|^{p}+W\left(\underline{u}\left(x^{\prime}\right)\right) d x^{\prime} d x_{N} \\
= & \int_{U} \frac{1}{p}\left|\nabla\left(\underline{u}\left(x^{\prime}\right)\right)\right|^{p}+W\left(\underline{u}\left(x^{\prime}\right)\right) d x .
\end{aligned}
$$

Analogously,

$$
\begin{aligned}
Q \int_{U} & \frac{1}{p}|\nabla(\bar{u}+\phi)|^{p}+W(\bar{u}+\phi) d x \\
& \geqslant \int_{U} \frac{1}{p}\left|\nabla\left(\bar{u}\left(x^{\prime}\right)\right)\right|^{p}+W\left(\bar{u}\left(x^{\prime}\right)\right) d x
\end{aligned}
$$

for any $\phi \in C_{0}^{\infty}(U)$.

Now let $\Omega$ and $\eta$ be as in the claim of Proposition 2.3. Given a bounded domain $U \subset \mathbb{R}^{N}$ and $v \in W^{1, p}(U)$, we define

$$
E_{U}(v):=\int_{U \cap \Omega} \frac{1}{p}|\nabla v|^{p}+W(v) d x .
$$

We also denote by $\chi_{S}$ the characteristic function of a set $S$ and

$$
\begin{aligned}
\alpha & :=(u+\eta) \chi_{\{u+\eta>\bar{u}\}}+\bar{u} \chi_{\{u+\eta \leqslant \bar{u}\}}, \\
\beta & :=\bar{u} \chi_{\{u+\eta>\bar{u}\}}+(u+\eta) \chi_{\{\underline{u} \leqslant u+\eta \leqslant \bar{u}\}}+\underline{u} \chi_{\{u+\eta<\underline{u}\}}, \\
\gamma & :=(u+\eta) \chi_{\{u+\eta<\underline{u}\}}+\underline{u} \chi_{\{u+\eta \geqslant \underline{u}\}} .
\end{aligned}
$$


Notice that $\underline{u}\left(x^{\prime}\right) \leqslant \beta\left(x^{\prime}, x_{N}\right) \leqslant \bar{u}\left(x^{\prime}\right)$ for any $\left(x^{\prime}, x_{N}\right) \in \mathbb{R}^{N}$ and that $\beta$ agrees with $u$ outside $\Omega$. So, by Lemma 2.2 ,

$$
E_{\Omega}(\beta) \geqslant E_{\Omega}(u) .
$$

Moreover, the set $\{u+\eta>\bar{u}\}$ is contained in $\Omega$, thence it is bounded, and $\alpha=\bar{u}$ outside $\{u+\eta>\bar{u}\}$. Consequently, by (2.15),

$$
Q E_{\{u+\eta>\bar{u}\}}(\alpha) \geqslant E_{\{u+\eta>\bar{u}\}}(\bar{u}) .
$$

Analogously,

$$
Q E_{\{u+\eta<\underline{u}\}}(\gamma) \geqslant E_{\{u+\eta<\underline{u}\}}(\underline{u}) .
$$

By collecting the above estimates, we gather that

$$
\begin{aligned}
Q E_{\Omega}(u+\eta)= & Q E_{\{u+\eta>\bar{u}\}}(u+\eta) \\
& \quad+Q E_{\{\underline{u} \leqslant u \leqslant \bar{u}\}}(u+\eta)+Q E_{\{u+\eta<\underline{u}\}}(u+\eta) \\
= & Q E_{\{u+\eta>\bar{u}\}}(\alpha)+Q E_{\{\underline{u} \leqslant u \leqslant \bar{u}\}}(\beta)+Q E_{\{u+\eta<\underline{u}\}}(\gamma) \\
\geqslant & E_{\{u+\eta>\bar{u}\}}(\bar{u})+E_{\{\underline{u} \leqslant u \leqslant \bar{u}\}}(\beta)+E_{\{u+\eta<\underline{u}\}}(\underline{u}) \\
= & E_{\Omega}\left(\bar{u} \chi_{\{u+\eta>\bar{u}\}}+\beta \chi_{\{\underline{u} \leqslant u \leqslant \bar{u}\}}+\underline{u} \chi_{\{u+\eta<\underline{u}\}}\right) \\
= & E_{\Omega}(\beta) \\
\geqslant & E_{\Omega}(u),
\end{aligned}
$$

as desired.

2.8. Proof of Lemma 2.4. By direct methods, we take $\beta_{R}$ to be the minimizer of

$$
\mathcal{J}_{R}(v):=\int_{B_{R}^{N}} \frac{1}{p}|\nabla v|^{p}+\tilde{W}(v) d x
$$

over functions $v \in W^{1, p}\left(B_{R}^{N}\right)$ with trace -1 on $\partial B_{R}^{N}$. By (2.2) and (2.3), we may suppose $\left|\beta_{R}\right| \leqslant 1$, and, in fact, $\left|\beta_{R}\right|<1$ by Lemma 2.1 .

Thus, it only remains to prove (2.6). If, by contradiction, (2.6) were false, we would have $\beta_{R} \leqslant a$ for infinitely many $R$ 's, for a suitable $a<1$. Hence, from (2.4),

$$
\tilde{W}\left(\beta_{R}(x)\right) \geqslant \inf _{(-\infty, a]} \tilde{W}=: \alpha>0
$$

for any $x$, for infinitely many $R$ 's, and so

$$
\mathcal{J}_{R}\left(\beta_{R}\right) \geqslant \operatorname{const} \alpha R^{N} \text {. }
$$

On the other hand, if we take $w$ to be -1 on $\partial B_{R}^{N}$ and +1 in $B_{R-1}^{N}$, we may achieve the bound

$$
\mathcal{J}_{R}(w) \leqslant \operatorname{const} R^{N-1},
$$

due to (2.3).

The minimality of $\beta_{R}$ is in contradiction with (2.16) and (2.17), thus proving (2.6). 
2.9. Proof of Lemma 2.5. We may suppose that

$$
\inf _{\mathbb{R}^{N}} v>-1
$$

otherwise we are done.

We claim that for any $\epsilon>0$ and $\rho>0$,

$$
\begin{aligned}
& \text { there exists } \bar{x}=\bar{x}(\epsilon, \rho) \in \mathbb{R}^{N} \text { such that } \\
& v(x) \geqslant 1-\epsilon \text { for any } x \in B_{\rho}^{N}(\bar{x}) .
\end{aligned}
$$

For this, making use of (2.7), we let $x_{j} \in \mathbb{R}^{N}$ be a sequence such that $v\left(x_{j}\right)$ approaches 1 as $j \rightarrow+\infty$. Then, by the regularity estimates of [DiB83, Tol84], if $w_{j}(x):=v\left(x+x_{j}\right)$ we have that, up to a subsequence, $w_{j}$ converges locally uniformly to some $w$ which is a weak solution of (1.4) and so that $w(0)=1$.

By Lemma 2.1, $w$ must be identically 1. Since $w_{j}$ tends to $w$ uniformly in $B_{\rho}^{N}(0)$, we take $j_{\epsilon, \rho}$ so that

$$
\left\|w_{j}-w\right\|_{L^{\infty}\left(B_{\rho}^{N}(0)\right)} \leqslant \epsilon
$$

for any $j \geqslant j_{\epsilon, \rho}$ and we set $\bar{x}:=x_{j_{\epsilon, \rho}}$.

Then,

$$
|v(x)-1|=\left|w_{j_{\epsilon, \rho}}(x-\bar{x})-1\right| \leqslant\left\|w_{j_{\epsilon, \rho}}-w\right\|_{L^{\infty}\left(B_{\rho}^{N}(0)\right)} \leqslant \epsilon
$$

for any $x \in B_{\rho}^{N}(\bar{x})$, proving (2.19).

We now consider the barrier in Lemma 2.4, For this scope, we use (2.18) to choose

$$
\lambda:=\frac{1}{2}\left(\inf _{\mathbb{R}^{N}} v-1\right) \in(-1,0]
$$

and to note that

$$
v \text { is also a weak solution of (2.5), }
$$

because of (2.3). Then, we set $\beta_{R}(x ; \tilde{x}):=\beta_{R}(x-\tilde{x})$ for any $\tilde{x} \in \mathbb{R}^{N}$.

If we take

$$
\epsilon:=1-\sup _{B_{1}^{N}(0)} \beta_{1}>0
$$

and $\bar{x}=\bar{x}(\epsilon, 2)$ in (2.19) , we deduce that $v(x) \geqslant \beta_{1}(x ; \bar{x})$ for any $x \in B_{1}^{N}(\bar{x})$.

By enlarging $R$ and sliding $\beta_{R}$, Lemma 2.1, (2.6) and (2.20) imply the desired claim.

2.10. Proof of Corollary 2.6. We have that

$$
\sup _{\mathbb{R}^{N}} v=1
$$

Indeed, if not, we would have $a_{0} \leqslant v \leqslant a_{1}$, with $-1<a_{0} \leqslant a_{1}<1$, due to (2.9), and so

$$
E_{R}(v) \geqslant \int_{B_{R}^{N}} W(v) d x \geqslant \operatorname{const} R^{N} \inf _{\left[a_{0}, a_{1}\right]} W
$$

for any $R>0$, in contradiction with (2.10).

Then, the result follows from Lemma 2.5 . 
2.11. Proof of Lemma 2.7. First of all, we observe that, for any $\vartheta>0$, there cannot be more than

$$
\text { two points in }\{W=\vartheta\} \cap[-1,1] \text {. }
$$

Indeed, if, say $W\left(r_{1}\right)=W\left(r_{2}\right)=W\left(r_{3}\right)=\vartheta$ with $-1 \leqslant r_{1}<r_{2}<r_{3} \leqslant 1$, Rolle's Theorem yields that there would exist $s_{1} \in\left(r_{1}, r_{2}\right) \subseteq(-1,1)$ and $s_{2} \in\left(r_{2}, r_{3}\right) \subseteq$ $(-1,1)$ such that $W^{\prime}\left(s_{1}\right)=W^{\prime}\left(s_{2}\right)=0$; hence $s_{1}=s_{2}=\kappa$, which gives the contradiction that proves (2.21).

Now, we take $h$ as in the statement of Lemma 2.7 We recall that, thanks to Corollary 4.8 of [FSV08, we have

$$
\frac{p-1}{p}\left|h^{\prime}(\tau)\right|^{p}-W(h(\tau))=\frac{p-1}{p}\left|h^{\prime}(\sigma)\right|^{p}-W(h(\sigma))
$$

for any $\tau, \sigma \in \mathbb{R}$.

Moreover, by Lemma 4.10 in [FSV08, we have that one of the following possibilities holds:

I. $h$ is constant,

II. $\left\{h^{\prime}=0\right\}=\emptyset$,

III. $h^{\prime}(t) \neq 0$ for any $t$ in a bounded interval of the form $\left(\beta_{1}, \beta_{2}\right)$ with $h^{\prime}\left(\beta_{1}\right)=$ $h^{\prime}\left(\beta_{2}\right)=0$ and

$$
W\left(h\left(\beta_{1}\right)\right)=W\left(h\left(\beta_{2}\right)\right)=W\left(\inf _{\mathbb{R}} h\right)=W\left(\sup _{\mathbb{R}} h\right) .
$$

IV. $h^{\prime}(t) \neq 0$ for any $t$ in an unbounded interval either of the form $(\beta,+\infty)$ or $(-\infty, \beta)$, with $\beta \in \mathbb{R}, h^{\prime}(\beta)=0$ and

$$
W(h(\beta))=W\left(\inf _{\mathbb{R}} h\right)=W\left(\sup _{\mathbb{R}} h\right) .
$$

Let us suppose that case I holds. Then, $h$ is identically equal to some $c$, and, by (2.11), we have that $W^{\prime}(c)=0$. Accordingly, $c \in\{-1, \kappa,+1\}$ and we are in (P1) of Lemma 2.7.

Suppose now that case II holds; hence $h$ is monotone and bounded. Let

$$
\ell_{ \pm}:=\lim _{t \rightarrow \pm \infty} h(t) \text {. }
$$

Note that

$$
\ell_{-}<\ell_{+} \text {. }
$$

Also, by (2.11) and the regularity results of DiB83, Tol84, we have that

$$
\lim _{t \rightarrow \pm \infty} h^{\prime}(t)=0
$$

and that

$$
W^{\prime}\left(\ell_{ \pm}\right)=0
$$

Therefore,

$$
\ell_{-}, \ell_{+} \in\{-1, \kappa,+1\}
$$

We claim that

$$
\ell_{-} \neq \kappa \text { and } \ell_{+} \neq \kappa \text {. }
$$

Indeed, suppose, by contradiction, that $\ell_{-}=\kappa$ (the case $\ell_{+}=\kappa$ may be ruled out in the same way). Then, by (2.25) and (2.27), we have that $\ell_{+}=+1$. 
Thence, making use of (2.22) and (2.26), we have

$$
\begin{aligned}
0=-W\left(\ell_{+}\right)=\lim _{\tau \rightarrow+\infty} \frac{p-1}{p}\left|h^{\prime}(\tau)\right|^{p}-W(h(\tau)) \\
=\lim _{\sigma \rightarrow-\infty} \frac{p-1}{p}\left|h^{\prime}(\sigma)\right|^{p}-W(h(\sigma))=-W(\kappa)<0 .
\end{aligned}
$$

This contradiction proves (2.28).

Then, case II, (2.27) and (2.28) say that we are in case (P2) of Lemma 2.7

Let us now deal with case III. We set $b_{i}:=h\left(\beta_{i}\right)$. Note that $b_{1} \neq b_{2}$, because $h$ is strictly monotone in $\left(\beta_{1}, \beta_{2}\right)$. For definiteness, we thus assume, without loss of generality, that

$$
b_{1}<b_{2} \text {. }
$$

Since $h(t) \in[-1,1]$ for any $t \in \mathbb{R}$, the use of Lemma 2.1 gives that

$$
\left|b_{i}\right|<1 \text {. }
$$

We now define

$$
\vartheta_{*}:=W\left(\inf _{\mathbb{R}} h\right)
$$

We observe that, by (2.23),

$$
\vartheta_{*}=W\left(\inf _{\mathbb{R}} h\right)=W\left(\sup _{\mathbb{R}} h\right)=W\left(b_{1}\right)=W\left(b_{2}\right)
$$

and therefore, by (2.30), $\vartheta_{*}>0$. Consequently, from (2.21) and (2.29),

$$
b_{1}=\inf _{\mathbb{R}} h \text { and } b_{2}=\sup _{\mathbb{R}} h,
$$

which says that such an infimum and supremum are attained.

Hence, being in case III with (2.30) and (2.31), we have reduced the situation to case (P3) of Lemma 2.7

In order to complete the proof of Lemma 2.7, we now show that case IV cannot hold.

We argue by contradiction. If case IV held, we may suppose, without loss of generality, that

$$
h^{\prime}(t)>0 \text { for } t>\beta \text {. }
$$

Note that $|h(\beta)|<1$ due to Lemma 2.1, and so

$$
W(h(\beta))>0 .
$$

Also let

$$
\ell:=\lim _{t \rightarrow+\infty} h(t)
$$

By (2.33), we have that

$$
\ell>h(\beta) .
$$

Also, (2.11) and the regularity results of [DiB83, Tol84] yield that

$$
\lim _{t \rightarrow \pm \infty} h^{\prime}(t)=0
$$

and

$$
W^{\prime}(\ell)=0
$$


Therefore, recalling (2.22), we obtain

$$
\begin{array}{r}
-W(\ell)=\lim _{\tau \rightarrow+\infty}\left(\frac{p-1}{p}\left|h^{\prime}(\tau)\right|^{p}-W(h(\tau))\right) \\
=\frac{p-1}{p}\left|h^{\prime}(\beta)\right|^{p}-W(h(\beta))=-W(h(\beta)) .
\end{array}
$$

As a consequence, using (2.35) and Rolle's Theorem, we see that there exists $s \in$ $(h(\beta), \ell) \subseteq(-1,1)$ for which $W^{\prime}(s)=0$. By our assumption on the potential, this gives that $s=\kappa$ and so $h(\beta)<\kappa<\ell$.

Hence, by (2.36), $\ell=+1$ and so a contradiction easily follows from (2.34) and (2.37).

This contradiction proves (2.32) and finishes the proof of Lemma 2.7

2.12. Proof of Corollary 2.8. If (S2) in Corollary 2.8 holds, then (P1) in Lemma 2.7 cannot hold. So either (P2) or (P3) holds, due to Lemma 2.7.

If (P2) holds, then (S2) is obvious.

Suppose now, by contradiction, that (P3) holds and (S2) does not hold. Without loss of generality, we may then suppose that $h(t) \geqslant \kappa$ for any $\kappa \in \mathbb{R}$. Thus, if we set $b_{i}:=h\left(\beta_{i}\right)$ for $i=1,2$, we get that $W\left(b_{1}\right)=W\left(b_{2}\right)$, with $b_{1}, b_{2} \in[\kappa, 1)$, in contradiction with our shape assumption on $W$.

2.13. Proof of Lemma 2.9. Suppose that condition (a) in the statement of Lemma 2.9 holds. Our purpose is to show that (b) holds, i.e. that (1.6) is satisfied.

If $h$ is constantly equal to +1 or -1 , its energy vanishes and (1.6) trivially holds. Thus, we just need to prove that if $h$ is strictly monotone with limits \pm 1 , then (1.6) holds.

We take $v$ to agree with $h$ outside $[-R, R]$. By an easy density argument, we may suppose that

$$
v \in C^{1}(\mathbb{R}) .
$$

Also, without loss of generality, we may also assume that

$$
h^{\prime}>0
$$

and that $R$ is so large that $h(R)$ (resp., $h(-R)$ ) is very close to +1 (resp., -1 ), and so

$$
v(R)=h(R)>h(-R)=v(-R) .
$$

We denote by $q$ the conjugate exponent of $p$, namely $q:=p /(p-1)$, and, for any $\theta \in \mathbb{R}$, we define

$$
G(\theta):=\int_{-1}^{\theta}(q W(s))^{1 / q} d s .
$$

From (2.38) and Young's Inequality,

$$
\begin{aligned}
\left|\frac{d}{d t} G(v(t))\right| & =\left|v^{\prime}(t)\right|(q W(v(t)))^{1 / q} \\
& \leqslant \frac{1}{p}\left|v^{\prime}(t)\right|^{p}+W(v(t)) .
\end{aligned}
$$


As a consequence, recalling (2.40),

$$
\begin{aligned}
& G(v(R))-G(v(-R))=|G(v(R))-G(v(-R))| \\
& \quad=\left|\int_{-R}^{R} \frac{d}{d t} G(v(t)) d t\right| \leqslant \int_{-R}^{R} \frac{1}{p}\left|v^{\prime}(t)\right|^{p}+W(v(t)) d t .
\end{aligned}
$$

Moreover, by Corollary 4.9 in FSV08,

$$
\frac{p-1}{p}\left|h^{\prime}(t)\right|^{p}-W(h(t))=0
$$

for any $t \in \mathbb{R}$, and so, recalling (2.39),

$$
\frac{1}{p}\left(h^{\prime}(t)\right)^{p}+W(h(t))=(q W(h(t)))^{1 / q} h^{\prime}(t) .
$$

As a consequence, by using again (2.39) to change the variable of integration,

$$
\begin{gathered}
\int_{-R}^{R} \frac{1}{p}\left(h^{\prime}(t)\right)^{p}+W(h(t)) d t=\int_{-R}^{R}(q W(h(t)))^{1 / q} h^{\prime}(t) d t \\
=\int_{h(-R)}^{h(R)}(q W(\tau))^{1 / q} d \tau=G(v(R))-G(v(-R)) .
\end{gathered}
$$

This and (2.41) show that $h$ satisfies (1.6).

The above arguments have proven that condition (a) in the statement of Lemma 2.9 implies condition (b).

Vice versa, suppose that (b) holds.

Then,

$$
h \text { cannot be constantly equal to } \kappa \text {. }
$$

Indeed, if $h$ were constantly equal to $\kappa$, we would let $v_{R}$ be such that $v_{R}(t)=1$ for any $|t| \leqslant R, v_{R}(t)=\kappa$ for any $|t| \geqslant R+1$ and $\left|v_{R}^{\prime}\right| \leqslant 2$. Then,

$$
\begin{aligned}
0 & \leqslant \int_{-(R+1)}^{R+1} \frac{1}{p}\left(\left|v_{R}^{\prime}\right|^{p}-\left|h^{\prime}\right|^{p}\right)+W\left(v_{R}\right)-W(h) d t \\
& \leqslant \frac{2^{p+1}}{p}+2\|W\|_{L^{\infty}([-1,1])}-2(R+1) W(\kappa),
\end{aligned}
$$

by virtue of (1.6). A contradiction is then obtained by taking $R$ sufficiently large, recalling that $W(\kappa)>0$. This proves (2.42).

Moreover,

$$
\sup _{\mathbb{R}}|h|=+1
$$

Otherwise, we would have that $h(t) \in[a, b]$ for any $t \in \mathbb{R}$, with $-1<a \leqslant b<1$ and so

$$
\int_{-(R+1)}^{R+1} \frac{1}{p}\left|h^{\prime}(t)\right|^{p}+W(h(t)) d t \geqslant 2(R+1) \inf _{[a, b]} W,
$$

while, if $w_{R}$ is such that $w_{R}(t)=1$ for any $|t| \leqslant R, w_{R}(t)=h(t)$ for any $|t| \geqslant R+1$ and $\left|w_{R}^{\prime}\right| \leqslant 2$, then

$$
\int_{-(R+1)}^{R+1} \frac{1}{p}\left|w_{R}^{\prime}(t)\right|^{p}+W\left(w_{R}(t)\right) d t \leqslant \frac{2^{p+1}}{p}+2\|W\|_{L^{\infty}([-1,1])} .
$$


From (1.6), (2.44) and (2.45), a contradiction easily follows by taking $R$ large, and this proves (2.43).

Notice in particular that (2.43) implies that case (P3) in Lemma 2.7 cannot hold. This observation, (2.42) and the classification in Lemma 2.7 imply that (a) holds, thus ending the proof of Lemma 2.9.

Though we do not need it here, we would like to remark that our proof of Lemma 2.9 also shows that monotone $1 \mathrm{D}$ solutions with limits -1 and +1 are minimizing among $1 \mathrm{D}$ functions that have the same limits.

2.14. Proof of Proposition 2.10. We set

$$
c_{u}:=\sup _{r \in\left[\inf _{\mathbb{R}^{N}} u, \sup _{\mathbb{R}^{N}} u\right]}(-W(r))=-\inf _{r \in\left[\inf _{\mathbb{R}^{N}} u, \sup _{\mathbb{R}^{N}} u\right]} W(r) .
$$

Since the roles of $\underline{u}$ and $\bar{u}$ are symmetrical in the statement of Proposition 2.10, we may assume for definiteness that

$$
W\left(\inf _{\mathbb{R}} \underline{u}\right) \leqslant W\left(\sup _{\mathbb{R}} \bar{u}\right),
$$

so that, by Lemma 4.13 in FSV08, formula (4.28) of [FSV08, holds true.

Consequently, we may apply Lemma 4.14 in FSV08 and obtain that $\underline{u}$ must satisfy one of the following possibilities:

A. $\underline{u}$ is constant.

B. $\left\{\underline{u}^{\prime}=0\right\}=\emptyset$.

C. There exist $\beta \in \mathbb{R}$ in such a way that $\underline{u}^{\prime}(t)<0$ for $t<\beta$ and $\underline{u}(t)=\inf _{\mathbb{R}} \underline{u}$ for $t \geqslant \beta$.

D. There exist $\beta \in \mathbb{R}$ in such a way that $\underline{u}^{\prime}(t)>0$ for $t>\beta$ and $\underline{u}(t)=\inf _{\mathbb{R}} \underline{u}$ for $t \leqslant \beta$.

E. There exist $\beta_{1} \leqslant \beta_{2} \in \mathbb{R}$ in such a way that $\underline{u}^{\prime}(t)<0$ for $t<\beta_{1}, \underline{u}^{\prime}(t)>0$ for $t>\beta_{2}$ and $\underline{h}(t)=\inf _{\mathbb{R}} \underline{u}$ for $t \in\left[\beta_{1}, \beta_{2}\right]$.

In fact, cases $\mathrm{C}, \mathrm{D}$ and $\mathrm{E}$ cannot hold here, due to Lemma 2.7 Thus,

$$
\text { either } \underline{u} \text { is constant or }\left\{\underline{u}^{\prime}=0\right\}=\emptyset \text {. }
$$

We claim that

$$
\underline{u} \text { is not constantly equal to } \kappa \text {. }
$$

Indeed, otherwise, by (2.47),

$$
W(\kappa) \leqslant W\left(\sup _{\mathbb{R}} \bar{u}\right)
$$

and so, by our hypotheses on $W$,

$$
\sup _{\mathbb{R}} \bar{u}=\kappa .
$$

This is in contradiction with (1.2) and it thus proves (2.49).

By force of Lemma 2.7 (1.2), (2.48) and (2.49), we have thus proven that the claim in $(\mathrm{C} 1)$ of Proposition 2.10 holds true. 
We now use this information to prove (C2). For this purpose, we first show that

$$
\sup _{\mathbb{R}} \bar{u}=+1 .
$$

Indeed, if the contrary were true, (C1) and (1.2) would imply that $\underline{u}$ is constantly equal to -1 and therefore

$$
\inf _{\mathbb{R}^{N}} u=-1 \text { and } \sup _{\mathbb{R}^{N}} u<1 .
$$

Thus, by Lemma 2.5 (applied to $v:=-u$ ), we would have that $u$ is constant, in contradiction with (1.2).

This proves (2.50), which, together with Lemma 2.7, gives that the claim in (C2) of Proposition 2.10 holds true.

Notice that (C1), (C2) and Lemma 2.9 imply that both $\underline{u}$ and $\bar{u}$ satisfy (1.6).

We now check the energy bound in (2.12). We prove the bound for $\underline{u}$, since the one for $\bar{u}$ is analogous.

For this, we observe that $(\mathrm{C} 1),(\mathrm{C} 2)$ and (2.46) imply that $c_{u}=0$. Thus, we exploit Corollary 4.16 of [FSV08] to conclude that

$$
\text { const } \begin{aligned}
R^{N-1} & \geqslant \int_{B_{R}^{N}} \frac{1}{p}|\nabla \underline{u}|^{p}+W(\underline{u})+c_{u} d x \\
& =\int_{B_{R}^{N}} \frac{1}{p}|\nabla \underline{u}|^{p}+W(\underline{u}) d x .
\end{aligned}
$$

From this, we obtain (2.12) and we end the proof of Proposition 2.10.

2.15. Proof of Proposition 2.11. We first show that (2.13) implies that $\underline{u}$ is 1D. In the course of this proof, to match with the notation in FSV08, we set $\lambda_{1}(t):=$ $(p-1) t^{p-2}$ and $\lambda_{2}(t):=t^{p-2}$. The tangential gradient of a smooth function $\psi$ along any regular level set of a smooth function $v$ will be denoted by $\nabla_{L} \psi$ (see (2.6) in FSV08 for further details). The sum of the squares of the principal curvatures of any regular level sets of $v$ will be denoted by $\mathcal{K}_{v}^{2}$ (see Section 2.3 of [FSV08] for additional comments).

If we define $Y\left(x^{\prime}\right):=\left(x^{\prime}, \underline{u}\left(x^{\prime}\right)\right)$, we deduce from (2.13) that

$$
\int_{\substack{x^{\prime} \in \mathbb{R} N-1 \\\left|Y\left(x^{\prime}\right)\right| \leqslant R}}\left|\nabla \underline{u}\left(x^{\prime}\right)\right|^{p} d x^{\prime} \leqslant \text { const } R^{2} .
$$

This and Lemma 5.1 in [FSV08 imply that

$$
\int_{\sqrt{R} \leqslant\left|Y\left(x^{\prime}\right)\right| \leqslant R} \frac{\left|\nabla \underline{u}\left(x^{\prime}\right)\right|^{p}}{\left|Y\left(x^{\prime}\right)\right|^{2}} d x^{\prime} \leqslant \text { const } \ln R,
$$

as long as $R$ is appropriately large.

We now define

$$
\phi\left(x^{\prime}\right):=\left\{\begin{array}{cc}
1 & \text { if }\left|Y\left(x^{\prime}\right)\right| \leqslant \sqrt{R}, \\
\frac{2 \ln \left(R / Y\left(x^{\prime}\right) \mid\right)}{\ln R} & \text { if } \sqrt{R}<\left|Y\left(x^{\prime}\right)\right|<R, \\
0 & \text { if }\left|Y\left(x^{\prime}\right)\right| \geqslant R .
\end{array}\right.
$$

\footnotetext{
${ }^{4}$ Notice that, at this level of the proof, we cannot obtain (C2) just by exchanging $\bar{u}$ and $\underline{u}$, since their role is fixed by (2.47).
} 
We observe that

$$
\left|\nabla \phi\left(x^{\prime}\right)\right| \leqslant \text { const } \frac{\left|\left(1, \nabla \underline{u}\left(x^{\prime}\right)\right)\right|}{\left|Y\left(x^{\prime}\right)\right| \ln R} \leqslant \frac{\text { const }}{\left|Y\left(x^{\prime}\right)\right| \ln R}
$$

for any $x^{\prime} \in \mathbb{R}^{N-1}$ such that $\sqrt{R}<\left|Y\left(x^{\prime}\right)\right|<R$.

Therefore, exploiting (2.51) above and (4.17) of [FSV08, we conclude 5 that

$$
\begin{aligned}
& \int_{B_{\sqrt{R} / 2}^{N-1} \cap\{\nabla \underline{u} \neq 0\}}\left(\left.\lambda_{1}\left|\nabla_{L}\right| \nabla \underline{u}\right|^{2}+\lambda_{2}|\nabla \underline{u}|^{2} \mathcal{K}_{\underline{u}}^{2}\right) d x^{\prime} \\
\leqslant & \int_{\mathbb{R}^{N-1} \cap\{\nabla \underline{u} \neq 0\}}\left(\lambda_{1}\left|\nabla_{L}\right| \nabla \underline{u}||^{2}+\lambda_{2}|\nabla \underline{u}|^{2} \mathcal{K}_{\underline{u}}^{2}\right) \phi^{2} d x^{\prime} \\
\leqslant & \operatorname{const} \int_{\mathbb{R}^{3}}|\nabla \underline{u}|^{p}|\nabla \phi|^{2} d x^{\prime} \\
\leqslant & \frac{\operatorname{const}}{\ln ^{2} R} \int_{\sqrt{R} \leqslant\left|Y\left(x^{\prime}\right)\right| \leqslant R} \frac{\left|\nabla \underline{u}\left(x^{\prime}\right)\right|^{p}}{\left|Y\left(x^{\prime}\right)\right|^{2}} d x^{\prime} \\
\leqslant & \frac{\operatorname{const}}{\ln R},
\end{aligned}
$$

for $R$ suitably large. Here the above $\lambda_{i}$ is short for $\lambda_{i}(|\nabla \underline{u}|)$.

Consequently, by taking $R$ arbitrarily large, we obtain that both $\nabla_{L}|\nabla \underline{u}|$ and $\mathcal{K}_{\underline{u}}$ vanish on $\{\nabla \underline{u} \neq 0\}$ and so, by Lemma 2.11 of [FSV08, $\underline{u}$ is $1 \mathrm{D}$.

Of course, by exchanging the roles of $\bar{u}$ and $\underline{u}$, we have also proven that

$$
\begin{aligned}
& \text { if } \int_{B_{R}^{N-1}}\left|\nabla \bar{u}\left(x^{\prime}\right)\right|^{p} d x^{\prime} \leqslant C R^{2}, \\
& \text { then } \bar{u} \text { is } 1 \mathrm{D} .
\end{aligned}
$$

We now prove that, if $N \leqslant 4$ and (2.14) holds, then $\bar{u}$ and $\underline{u}$ are both $1 \mathrm{D}$. In fact, since (2.14) is stronger than (2.13), we already know that $\underline{u}$ is $1 \mathrm{D}$, and we may therefore focus on proving that $\bar{u}$ is $1 \mathrm{D}$ too.

To this end, we exploit Lemma 4.1 of [FSV08] (alternatively, if $p=2$, the computations in Section 2 of [AC00] could also be employed). From such a result, (2.8), (2.14) and the fact that $N \leqslant 4$, we see that

$$
E_{R}\left(u_{t}\right) \leqslant \operatorname{const} R^{3},
$$

where $u_{t}(x):=u\left(x^{\prime}, x_{N}-t\right)$, for any $t \in \mathbb{R}$.

Consequently,

and this implies (2.52).

$$
E_{R}(\bar{u}) \leqslant \text { const } R^{3}
$$

Therefore, by (2.52), we conclude that $\bar{u}$ is $1 \mathrm{D}$, finishing the proof of Proposition 2.11 .

We remark that the above proof may be easily adapted to a more general framework, and it shows that any function with bounded gradient satisfying both the

${ }^{5}$ We recall that (4.17) of [FSV08] in this setting reads

$$
\begin{aligned}
& \int_{\mathbb{R}^{N-1} \cap\{\nabla \underline{u} \neq 0\}}\left(\lambda_{1}\left|\nabla_{L}\right| \nabla \underline{u}||^{2}+\lambda_{2}|\nabla \underline{u}|^{2} \mathcal{K}_{\underline{u}}^{2}\right) \phi^{2} d x^{\prime} \\
\leqslant & \int_{\mathbb{R}^{N-1} \cap\{\nabla \underline{u} \neq 0\}}|\nabla \underline{u}|^{p}\left(|\nabla \phi|^{2}+(p-2)\left(\frac{\nabla \underline{u}}{|\nabla \underline{u}|} \cdot \nabla \phi\right)^{2}\right) d x^{\prime} .
\end{aligned}
$$

Theorem 4.12 of FSV08 proves that such a formula holds for our solutions. 
geometric estimate in (4.17) of [FSV08, and the kinetic energy growth in (2.13) must be 1D.

2.16. Proof of Proposition 2.12, During this proof, we will use again the notation of $\lambda_{1}, \lambda_{2}, \nabla_{L} v$ and $\mathcal{K}_{v}$ stated on page 597 .

In order to prove Proposition 2.12, we suppose that $\underline{u}$ is $2 \mathrm{D}$ and we show that, in fact, it is 1D. To this extent, we use formula (4.17) of [FSV08] (see also the previous footnote 5) to get that

$$
\begin{aligned}
& \int_{\mathbb{R}^{N-1} \cap\{\nabla \underline{u} \neq 0\}}\left(\left.\lambda_{1}\left|\nabla_{L}\right| \nabla \underline{u}\right|^{2}+\lambda_{2}|\nabla \underline{u}|^{2} \mathcal{K}_{\underline{u}}^{2}\right) \varphi^{2} d x^{\prime} \\
\leqslant & \operatorname{const} \int_{\mathbb{R}^{N-1}}|\nabla \underline{u}|^{p}|\nabla \varphi|^{2} d x^{\prime}
\end{aligned}
$$

for any Lipschitz and compactly supported function $\varphi: \mathbb{R}^{N-1} \rightarrow \mathbb{R}$, where $\lambda_{i}$ is short for $\lambda_{i}(|\nabla \underline{u}|)$.

In fact, since we assumed $\underline{u}$ to be $2 \mathrm{D}$, up to a change of coordinates, we write $\underline{u}\left(x^{\prime}\right)=\underline{u}\left(x_{1}, x_{2}\right)$.

Therefore, supposing with no loss of generality that $N \geqslant 4$, we take an arbitrary Lipschitz and compactly supported function $\phi: \mathbb{R}^{2} \rightarrow \mathbb{R}$ and $\tau \in C_{0}^{\infty}\left(\mathbb{R}^{N-3}\right)$, with

$$
\int_{\mathbb{R}^{N-3}} \tau^{2}\left(x_{3}, \ldots, x_{N-1}\right) d x_{3} \ldots d x_{N-1}=1
$$

and we choose

$$
\varphi\left(x^{\prime}\right):=\phi\left(x_{1}, x_{2}\right) \theta^{(N-3) / 2} \tau\left(\theta x_{3}, \ldots, \theta x_{N-1}\right)
$$

as a test function, where $\theta \in(0,1)$ is a fixed parameter.

Then, the estimate in (2.53) implies that

$$
\begin{aligned}
& \int_{\mathbb{R}^{2} \cap\{\nabla \underline{u} \neq 0\}}\left(\left.\lambda_{1}\left|\nabla_{L}\right| \nabla \underline{u}\right|^{2}+\lambda_{2}|\nabla \underline{u}|^{2} \mathcal{K}_{\underline{u}}^{2}\right) \phi^{2}\left(x_{1}, x_{2}\right) d x_{1} d x_{2} \\
= & \theta^{N-3} \int_{\mathbb{R}^{N-1} \cap\{\nabla \underline{u} \neq 0\}}\left(\left.\lambda_{1}\left|\nabla_{L}\right| \nabla \underline{u}\right|^{2}+\lambda_{2}|\nabla \underline{u}|^{2} \mathcal{K}_{\underline{u}}^{2}\right) \phi^{2}\left(x_{1}, x_{2}\right) \tau^{2}\left(\theta x_{3}, \ldots, \theta x_{N-1}\right) d x^{\prime} \\
\leqslant & \mathrm{const} \int_{\mathbb{R}^{N-1}}|\nabla \underline{u}|^{p}\left(\theta^{N-3} \tau^{2}\left(\theta x_{3}, \ldots, \theta x_{N-1}\right)\left|\nabla \phi\left(x_{1}, x_{2}\right)\right|^{2}\right. \\
& \left.+\|\phi\|_{L^{\infty}\left(\mathbb{R}^{2}\right)}^{2} \theta^{N-1}\left|\nabla \tau\left(\theta x_{3}, \ldots, \theta x_{N-1}\right)\right|^{2}\right) d x^{\prime} \\
= & \mathrm{const} \int_{\mathbb{R}^{N-1}}|\nabla \underline{u}|^{p}\left(\tau^{2}\left(x_{3}, \ldots, x_{N-1}\right)\left|\nabla \phi\left(x_{1}, x_{2}\right)\right|^{2}\right. \\
& \left.+\|\phi\|_{L^{\infty}\left(\mathbb{R}^{2}\right)}^{2} \theta^{2}\left|\nabla \tau\left(x_{3}, \ldots, x_{N-1}\right)\right|^{2}\right) d x^{\prime} .
\end{aligned}
$$

Therefore, by taking $\theta$ as close as we wish to 0 , we conclude that

$$
\begin{aligned}
& \int_{\mathbb{R}^{2} \cap\{\nabla \underline{u} \neq 0\}}\left(\lambda_{1}\left|\nabla_{L}\right| \nabla \underline{u}||^{2}+\lambda_{2}|\nabla \underline{u}|^{2} \mathcal{K}_{\underline{u}}^{2}\right) \phi^{2} d x_{1} d x_{2} \\
\leqslant & \text { const } \int_{\mathbb{R}^{2}}|\nabla \underline{u}|^{p}|\nabla \phi|^{2} d x_{1} d x_{2} \\
\leqslant & \operatorname{const} \int_{\mathbb{R}^{2}}|\nabla \phi|^{2} d x_{1} d x_{2}
\end{aligned}
$$

for any Lipschitz and compactly supported function $\phi: \mathbb{R}^{2} \rightarrow \mathbb{R}$. 
As a consequence, by Corollary 2.6 of [FSV08, we conclude that $\nabla_{L}|\nabla \underline{u}|$ and $\mathcal{K}_{\underline{u}}$ vanish identically on $\{\nabla \underline{u} \neq 0\}$ and therefore, by Lemma 2.11 of [FSV08], that $\underline{u}$ is 1D. This gives the claim of Proposition 2.12 .

2.17. Proof of Proposition 2.13. The proof is by contradiction. Suppose that $\bar{u}$ is not constantly equal to +1 . Then, it is strictly monotone, thanks to Lemma 2.9.

Therefore, there exists $\vartheta \in(-1,1), \omega^{\prime} \in S^{N-2}$ and $t_{o} \in \mathbb{R}$ in such a way that $\bar{u}\left(x^{\prime}\right)$ is, respectively, strictly greater than or strictly less than $\vartheta$ when $\omega^{\prime} \cdot x^{\prime}>t_{o}$ or $\omega^{\prime} \cdot x^{\prime}<t_{o}$.

Up to translation, we will assume $t_{o}=0$.

From this and (1.2), we conclude that

$$
\{u=\vartheta\} \subseteq\left\{\left(\omega^{\prime}, 0\right) \cdot x \geqslant 0\right\} .
$$

For $\epsilon>0$, we now consider the rescaled function $u_{\epsilon}(x):=u(x / \epsilon)$.

By (2.54), we obtain

$$
\left\{u_{\epsilon}=\vartheta\right\} \subseteq\left\{\left(\omega^{\prime}, 0\right) \cdot x \geqslant 0\right\} .
$$

Due to Bou90, PV05, we know that $\left\{u_{\epsilon}=\vartheta\right\}$ approaches, locally uniformly, the boundary of a set $E$ which has minimal perimeter, and so, from (2.55),

$$
\partial E \subseteq\left\{\left(\omega^{\prime}, 0\right) \cdot x \geqslant 0\right\} .
$$

As a consequence of this and of Theorem 17.4 in Giu84, we thus infer that $\partial E$ is a hyperplane.

Thence, from Corollary 7 of [FV08, $u$ is 1D.

Therefore, by force of (2.54), we have that $\{u=\vartheta\}$ is a hyperplane normal to $\left(\omega^{\prime}, 0\right)$, that is,

for some $c_{o} \in \mathbb{R}$.

$$
\{u=\vartheta\}=\left\{\left(\omega^{\prime}, 0\right) \cdot x=c_{o}\right\}
$$

Accordingly, $u\left(c_{o} \omega^{\prime}, x_{N}\right)=\vartheta$ for any $x_{N} \in \mathbb{R}$, which is in contradiction with (1.2).

2.18. Proof of Proposition 2.14, By Proposition 2.10, $\bar{u}$ and $\underline{u}$ satisfy (1.6). Thus, $u$ satisfies (1.6), thanks to Proposition 2.3.

Then, the claim follows from Proposition 2.13.

\section{Proof of Theorem 1.1}

By means of Proposition 2.12, both $\bar{u}$ and $\underline{u}$ are 1D.

In fact, $\bar{u}$ is identically +1 and $\underline{u}$ is identically -1 , thanks to Proposition 2.14 and $u$ satisfies (1.6) because of Proposition 2.3.

Then, Theorem 1.4 and Lemma 9.1 of VSS06 imply that $u$ is $1 \mathrm{D}$, as long as $N \leqslant 8$.

\section{An alternative Proof of Theorem 1.1}

We would like to remark that another strategy would lead to the proof of Theorem 1.1 without using the calibration result in Lemma 2.2 ,

Indeed, Lemma 2.2 has been used in this paper to deduce Proposition 2.3 and the latter was used in the above proof of Theorem 1.1 that $u$ also satisfies (1.6).

But this latter property may also be obtained via the following argument, which does not use either Lemma 2.2 or Proposition 2.3 
Suppose, by contradiction, that there exists $R>0$ and $\eta \in C^{\infty}\left(B_{R}^{N}\right)$ for which the inequality in (1.6) is false.

By direct minimization we now take $w \in W^{1, p}\left(B_{R}^{N}\right)$ to attain the minimum of

$$
\int_{B_{R}^{N}} \frac{1}{p}|\nabla w|^{p}+W(w) d x
$$

under the trace condition that $w=u$ on $\partial B_{R}^{N}$.

Then, our contradictory assumption implies that

$$
\int_{B_{R}^{N}} \frac{1}{p}|\nabla w|^{p}+W(w) d x<\int_{B_{R}^{N}} \frac{1}{p}|\nabla u|^{p}+W(u) d x .
$$

Let $\tilde{w}(x):=\min \left\{w(x), \bar{u}\left(x^{\prime}\right)\right\}$, for any $x=\left(x^{\prime}, x_{N}\right) \in \mathbb{R}^{N}$. Since, by Proposition 2.10, we know that $\bar{u}$ satisfies (1.6), it follows that

$$
\begin{aligned}
& \int_{B_{R}^{N}} \frac{1}{p}|\nabla \tilde{w}|^{p}+W(\tilde{w}) d x \\
= & \int_{B_{R}^{N} \cap\{w \leqslant \bar{u}\}} \frac{1}{p}|\nabla w(x)|^{p}+W(w(x)) d x \\
& +\int_{B_{R}^{N} \cap\{w>\bar{u}\}} \frac{1}{p}\left|\nabla \bar{u}\left(x^{\prime}\right)\right|^{p}+W\left(\bar{u}\left(x^{\prime}\right)\right) d x \\
= & \int_{B_{R}^{N} \cap\{w \leqslant \bar{u}\}} \frac{1}{p}|\nabla w|^{p}+W(w) d x \\
& \quad \int_{-R}^{R} \frac{1}{p}\left|\nabla \bar{u}\left(x^{\prime}\right)\right|^{p}+W\left(\bar{u}\left(x^{\prime}\right)\right) d x^{\prime} d x_{N} \\
\leqslant & \int_{B_{R}^{N} \cap\{w \leqslant \bar{u}\}} \frac{1}{p}|\nabla w|^{p}+W(w) d x \\
& +\int_{-R}^{R} \int_{B_{R}^{N-1} \cap\left\{w\left(x^{\prime}, x_{N}\right)>\bar{u}\left(x^{\prime}\right)\right\}} \frac{1}{p}\left|w\left(x^{\prime}, x_{N}\right)\right|^{p}+W\left(w\left(x^{\prime}, x_{N}\right)\right) d x^{\prime} d x_{N} \\
= & \int_{B_{R}^{N}} \frac{1}{p}|\nabla w|^{p}+W(w) d x ;
\end{aligned}
$$

thence, possibly replacing $w$ with $\tilde{w}$, we may suppose that $w \leqslant \bar{u}$. Analogously, $w \geqslant \underline{u}$. Therefore,

$$
\underline{u}\left(x^{\prime}\right) \leqslant w(x) \leqslant \bar{u}\left(x^{\prime}\right)
$$

for any $x=\left(x^{\prime}, x_{N}\right) \in \mathbb{R}^{N}$.

We extend $w$ to agree with $u$ outside $B_{R}^{N}$ and we define

$$
u_{t}(x)=u_{t}\left(x^{\prime}, x_{N}\right)=u\left(x^{\prime}, x_{N}-t\right) .
$$

Then, by construction, $u_{t}$ is a weak solution of (1.4) in $\mathbb{R}^{N}$ and $w$ is a weak solution of (1.4) in $\mathbb{R}^{N} \backslash \partial B_{R}^{N}$. Also, $\underline{u}$ and $\bar{u}$ are weak solutions of (1.4) in $\mathbb{R}^{N-1}$ and thus in $\mathbb{R}^{N}$ (when taken to be constant functions in the $N$ th direction).

We observe that a stronger version of (4.2) holds, namely

$$
\underline{u}\left(x^{\prime}\right)<w(x)<\bar{u}\left(x^{\prime}\right)
$$


for any $x=\left(x^{\prime}, x_{n}\right) \in \mathbb{R}^{N}$. To prove (4.3) , suppose that, say, $\underline{u}\left(\bar{x}^{\prime}\right)=w\left(\bar{x}^{\prime}, \bar{x}_{N}\right)$ for some $\left(\bar{x}^{\prime}, \bar{x}_{N}\right) \in \mathbb{R}^{N}$.

Note that $w=u>\underline{u}$ on $\partial B_{R}^{N}$, due to (1.2), so $\left(\bar{x}^{\prime}, \bar{x}_{N}\right) \notin B_{R}^{N}$. Then, the assumptions of Lemma 2.1 are fulfilled, due to Proposition 2.10 and so $w$ and $\underline{u}$ are identically equal. In particular, $w=u=\underline{u}$ on $\partial B_{R}^{N}$, in contradiction with (1.2). This proves (4.3).

Since

$$
\lim _{t \rightarrow-\infty} u_{t}\left(x^{\prime}, x_{N}\right)=\bar{u}\left(x^{\prime}\right)>w\left(x^{\prime}\right)
$$

due to (4.3), we deduce that there exists $t^{\star} \leqslant 0$ in such a way that $u_{t}(x)>w(x)$ for any $t<t^{\star}$ and any $x \in \mathbb{R}^{N}, u_{t^{\star}}(x) \geqslant w(x)$ for any $x \in \mathbb{R}^{N}$ and $u_{t^{\star}}\left(x^{\star}\right)=w\left(x^{\star}\right)$ for a suitable $x^{\star} \in \mathbb{R}^{N}$.

We show that

$$
t^{\star}=0
$$

Suppose $t^{\star}<0$. Then,

$$
u_{t^{\star}}>u=w \text { on } \partial B_{R}^{N} \text {; }
$$

thus $x^{\star}$ cannot lie on $\partial B_{R}^{N}$. Therefore, recalling that $\left\{\nabla u_{t^{\star}}=0\right\}=\emptyset$ by (1.2), we deduce from Lemma 2.1 that $w$ agrees identically with $u_{t^{\star}}$, in contradiction with (4.6). This proves (4.5), which in turn shows that $u \geqslant w$.

By arguing in the same way, sending $t \rightarrow+\infty$ in (4.4) (and exchanging $\underline{u}$ and $\bar{u}$ when needed), we also see that $u \leqslant w$.

Therefore, $u=w$, in contradiction with (4.3).

This ends the alternative proof of Theorem 1.1

\section{Proof of Theorem 1.2}

We suppose, for definiteness, that $N=4$ and that $\bar{u}$ is $2 \mathrm{D}$. By force of Proposition 2.12, we know that

$$
\bar{u} \text { is, in fact, } 1 \mathrm{D} \text {. }
$$

Also, recalling that here $p=2$, a well-known consequence of (1.2) (see, e.g., Lemma 7.1 in [FSV08] for a general result) is that

$$
\int_{\mathbb{R}^{4}}|\nabla \phi|^{2}+W^{\prime \prime}(u) \phi^{2} d x \geqslant 0
$$

for any smooth and compactly supported $\phi: \mathbb{R}^{4} \rightarrow \mathbb{R}$.

Accordingly, from (1.5), the compactness results of [DiB83, Tol84] and the assumption that $W \in C^{2}(\mathbb{R})$, we conclude that

$$
\int_{\mathbb{R}^{4}}|\nabla \phi|^{2}+W^{\prime \prime}(\bar{u}) \phi^{2} d x \geqslant 0
$$

for any $\phi \in W^{1,2}\left(\mathbb{R}^{4}\right)$.

Thence, from (5.1),

$$
\int_{\mathbb{R}}\left|\phi^{\prime}(t)\right|^{2}+W^{\prime \prime}(\bar{u}(t)) \phi^{2}(t) d t \geqslant 0
$$

for any $\phi \in W^{1,2}(\mathbb{R})$.

We now observe that

$\bar{u}$ cannot be constantly equal to $\kappa$. 
The proof is by contradiction. If $\bar{u}$ is constantly equal to $\kappa$, then $u(x) \in[-1, \kappa]$ for any $x \in \mathbb{R}^{4}$ and so $\Delta u=W^{\prime}(u) \geqslant 0$ in $\mathbb{R}^{4}$.

Consequently, by parabolicity estimates (see, for instance, HKM06] or Theorem 9.11(b) of [Far07]),

$$
\int_{B_{R}^{4}}|\nabla u(x)|^{2} d x \leqslant \operatorname{const} R^{2} .
$$

Thus, $u$ must be 1D (see, e.g., Lemma 5.2 in FSV08 for a general result).

The fact that $u$ is $1 \mathrm{D}$, strictly monotone, and approaches $\kappa$ at infinity is in contradiction with Lemma 2.7. This proves (5.3).

We now claim that

$$
\bar{u} \text { is non-increasing or non-decreasing, with } \sup _{\mathbb{R}}|\bar{u}|=+1 \text {. }
$$

To check this, we argue by contradiction. If (5.4) were false, Lemma 2.7 and (5.3) would say that $\left|\bar{u}^{\prime}\right|>0$ in the interval $\left(\beta_{1}, \beta_{2}\right)$, with $\bar{u}^{\prime}\left(\beta_{1}\right)=\bar{u}^{\prime}\left(\beta_{2}\right)=0$.

Then, we define $\phi_{\star}:=\chi_{\left[\beta_{1}, \beta_{2}\right]} \bar{u}^{\prime}$ and we observe that, in $\left(\beta_{1}, \beta_{2}\right)$, we have that

$$
\left|\phi_{\star}\right|>0
$$

and

$$
\phi_{\star}^{\prime \prime}=W^{\prime \prime}(\bar{u}) \phi_{\star} .
$$

Also,

$$
\phi_{\star}^{\prime}\left(\beta_{1}-1\right)=\phi_{\star}\left(\beta_{1}-1\right)=0 .
$$

Integrating by parts and using (5.6), one sees that

$$
\int_{\mathbb{R}}\left|\phi_{\star}^{\prime}(t)\right|^{2}+W^{\prime \prime}(\bar{u}(t)) \phi_{\star}^{2}(t) d t=0 ;
$$

thus $\phi_{\star}$ minimizes the left hand side of (5.2).

As a consequence, (5.6) holds in the whole $\mathbb{R}$.

Therefore, recalling (5.7), Cauchy's uniqueness result for ODEs implies that $\phi_{\star}$ vanishes identically.

Since this is in contradiction with (5.5), we have proven (5.4).

From (5.4), we deduce that

$$
\int_{\mathbb{R}}\left|\bar{u}^{\prime}(t)\right| d t=\lim _{a \rightarrow+\infty}\left|\int_{-a}^{a} \bar{u}^{\prime}(t) d t\right|=\lim _{a \rightarrow+\infty}|\bar{u}(a)-\bar{u}(-a)| \leqslant 2 .
$$

In addition, from (5.4) and Corollary 4.9 in FSV08, we obtain that

$$
\frac{1}{2}\left|\bar{u}^{\prime}(t)\right|^{2}-W(\bar{u}(t))=0
$$

for any $t \in \mathbb{R}$. This and (5.8) imply that

$$
\begin{aligned}
\int_{\mathbb{R}} & \frac{1}{2}\left|\bar{u}^{\prime}(t)\right|^{2}+W(\bar{u}(t)) d t \\
\quad & =\int_{\mathbb{R}}\left|\bar{u}^{\prime}(t)\right|^{2} d t \\
& \leqslant\|\nabla u\|_{C^{1}\left(\mathbb{R}^{4}\right)} \int_{\mathbb{R}}\left|\bar{u}^{\prime}\left(x^{\prime}\right)\right| d x^{\prime} \\
& \leqslant \text { const. }
\end{aligned}
$$


Consequently,

$$
\int_{B_{R}^{3}} \frac{1}{2}|\nabla \bar{u}(t)|^{2}+W(\bar{u}(t)) d t \leqslant \operatorname{const} R^{2},
$$

and so Proposition 2.11 implies that $\underline{u}$ is also 1D.

Then, the claim of Theorem 1.2 is, in this case, a consequence of Theorem 1.1

\section{Proof of Theorem 1.3}

By (1.7) and Theorem 1.1 of [Far03, we have that either $\bar{u}$ is identically +1 or

$$
\underline{u} \text { is identically }-1 \text {. }
$$

Without loss of generality, we suppose that the latter situation happens.

Hence, from Lemma 4.1 of [FSV08],

$$
E_{R}\left(u_{t}\right) \leqslant E_{R}(\underline{u})+\operatorname{const} R^{N-1} \leqslant \operatorname{const} R^{N-1},
$$

where $u_{t}(x):=u\left(x_{1}, \ldots, x_{N}+t\right)$, for any $t \in \mathbb{R}$ and so

$$
E_{R}(\bar{u}) \leqslant \text { const } R^{N-1} .
$$

As a consequence, since $\bar{u}$ is $(N-1) \mathrm{D}$,

$$
\int_{B_{R}^{N-1}} \frac{1}{p}\left|\nabla \bar{u}\left(x^{\prime}\right)\right|^{p}+W\left(\bar{u}\left(x^{\prime}\right)\right) d x^{\prime} \leqslant \text { const } R^{N-2} .
$$

We also note that

$$
\inf _{\mathbb{R}^{N-1}} \bar{u}>-1
$$

because of (1.2) and (1.7).

We now apply Corollary 2.6. More precisely, we take $v$ to be $\bar{u}$ in Corollary 2.6, so $N$ in Corollary 2.6 is $N-1$ here. Then (2.9) and (2.10) hold true, thanks to (6.2) and (6.3). Hence, from Corollary 2.6, we gather that $\bar{u}$ is identically +1 .

Recalling (6.1), we obtain the first claim in Theorem [1.3, namely that $\bar{u}$ is identically +1 and $\underline{u}$ is identically -1 .

This and Lemma 9.1 of VSS06 imply that $u$ is minimal.

Then, the last claim in Theorem 1.3 follows from Theorem 1.4 in VSS06.

\section{Proof of Theorem 1.4}

Let $c$ be as in (1.7). There are two cases, either $c \geqslant \kappa$ or $c \leqslant \kappa$. We deal with the first case, the second one being analogous.

By our assumptions on $W$, we have that $W^{\prime}(r) \leqslant 0$ if $r \in[c, 1] \subseteq[\kappa, 1]$.

Also, by (1.7), we know that

$$
\bar{u}\left(x^{\prime}\right) \in[c, 1] \subseteq[\kappa, 1] \text { for any } x^{\prime} \in \mathbb{R}^{N-1} .
$$

The above observations give that

$$
\Delta_{p} \bar{u}=W^{\prime}(\bar{u}) \leqslant 0 .
$$

Then, parabolicity estimates (see, for instance, HKM06 or Theorem 9.11(b) of [Far07]) and either (1.8) or (1.9) imply that

$$
\int_{B_{R}^{N-1}}\left|\nabla \bar{u}\left(x^{\prime}\right)\right|^{p} d x^{\prime} \leqslant \text { const } R^{N-1-p} \leqslant \text { const } R^{2}
$$

for large $R$.

Therefore, by Proposition 2.11, $\bar{u}$ is 1D. 
This, (1.7), (7.1) and Corollary 2.8 imply that

$$
\bar{u} \text { is constantly equal to }+1 \text {. }
$$

We now claim that

$$
\underline{u} \text { is constantly }-1 \text {. }
$$

To prove (7.3) we need to distinguish between the case in which (1.8) holds and the case in which (1.9) holds.

If (1.8) holds, we use Lemma 4.1 of [FSV08] and (7.2) to get

$$
\begin{aligned}
& \int_{B_{R}^{N}}|\nabla \underline{u}|^{p}+W(\underline{u}) d x \\
& \quad \leqslant \int_{B_{R}^{N}}|\nabla \bar{u}|^{p}+W(\bar{u}) d x+\operatorname{const} R^{N-1} \\
& \quad=0+\operatorname{const} R^{3} .
\end{aligned}
$$

Then, Proposition 2.11 gives that $\underline{u}$ is $1 \mathrm{D}$.

Accordingly, Proposition 2.10 and (1.7) imply (7.3).

Let us now prove (7.3) under assumption (1.9). For this scope, we make use of (7.2) and of Lemma 2.5 (whose assumptions are fulfilled here since $p=2$ ), to see that

$$
\inf _{\mathbb{R}^{N}} u=-1,
$$

that is,

$$
\inf _{\mathbb{R}^{N-1}} \underline{u}=-1
$$

This and (1.7) imply that

$$
\sup _{\mathbb{R}^{N-1}} \underline{u}<+1 .
$$

Thus, applying Lemma 2.5 to $v:=-\underline{u}$, we obtain (7.3) in this case too.

Having completed the proof of (17.3), the proof of Theorem 1.4 is finished, thanks to (7.2), (7.3) and the results of VSS06] (see in particular Theorem 1.4 and Lemma 9.1 there).

\section{Proof of Theorem 1.5}

First, we show that

$$
\bar{u} \text { is } 1 \mathrm{D} \text {. }
$$

To this extent, we use (1.2) and the assumption that $\{u=\kappa\}$ is a complete graph to obtain that $\bar{u} \geqslant \kappa$ and so

$$
\Delta_{p} \bar{u}=W^{\prime}(\bar{u}) \leqslant 0 .
$$

Consequently, by $p$-parabolicity estimates (see, for instance, [HKM06] or Theorem 9.11(b) of [Far07]),

$$
\int_{B_{R}^{N-1}}\left|\nabla \bar{u}\left(x^{\prime}\right)\right|^{p} d x^{\prime} \leqslant \text { const } R^{N-1-p}
$$

and so, by our assumptions on $p$,

$$
\int_{B_{R}^{N-1}}\left|\nabla \bar{u}\left(x^{\prime}\right)\right|^{p} d x^{\prime} \leqslant \text { const } R^{2}
$$


for any $R \geqslant 1$. This and Proposition 2.11 give (8.1). Analogously, we see that $\underline{u}$ is $1 \mathrm{D}$. Using the fact that $\{u=\kappa\}$ is a complete graph, (8.1) and Proposition 2.10, we deduce that $\bar{u}$ is constantly equal to +1 . Analogously, one proves that $\underline{u}$ is constantly equal to -1 .

Lemma 9.1 of VSS06 then yields the minimality of $u$ and, if $N \leqslant 8$, Theorem 1.4 of VSS06] gives that $u$ is $1 \mathrm{D}$.

\section{Proof of Proposition 1.6}

By taking limits in (1.6), we have that $\bar{u}$ is also a global (or class A, depending on the lingo) minimizer; thus it is $1 \mathrm{D}$, thanks to (1.10) above and Theorem $1.3 \mathrm{in}$ VSS06.

As a consequence, Lemma 2.9 gives that either $\bar{u}$ is constantly equal to +1 or -1 , or it is strictly monotone.

That $\bar{u}$ is constantly equal to -1 is ruled out by (1.2).

Also, $\bar{u}$ cannot be strictly monotone, due to Proposition 2.13 ,

Hence, $\bar{u}$ is constantly equal to +1 .

\section{Proof of Theorem 1.7}

By virtue of Proposition 1.6. we know that $\bar{u}$ is constantly equal to +1 and that $\underline{u}$ is constantly equal to -1 . Then, the claim of Theorem 1.7 follows from Theorem 1.4 of [VSS06].

\section{Proof of Theorem 1.8}

Without loss of generality, we suppose that (1.11) holds and that $u$ is not constant. Then, $\{u=0\} \neq \emptyset$ (see, e.g., Corollary 13 in [FV08 for a general result).

As a consequence, there exists $\alpha>0$ in such a way that $u\left(x^{\prime}, x_{N}\right) \geqslant 1 / 2$ for any $x^{\prime} \in \mathbb{R}^{N-1}$, as long as $x_{N} \geqslant \alpha$.

Thus, if $u_{\epsilon}(x):=u(x / \epsilon)$, we get that

$$
\left\{u_{\epsilon}=0\right\} \subseteq\left\{x_{N} \leqslant \epsilon \alpha\right\} .
$$

By Bou90, PV05, we know that $\left\{u_{\epsilon}=0\right\}$ approaches, locally uniformly, the boundary of a set $E$ with minimal perimeter.

From (11.1),

$$
\partial E \subseteq\left\{x_{N} \leqslant 1\right\}
$$

and so, by Theorem 17.4 of Giu84, we have that $\partial E$ is a hyperplane.

Thence, from Corollary 7 of [FV08, $u$ is 1D.

\section{Proof of Theorem 1.9}

By taking limits in (1.13), we have that $\bar{u}$ and $\underline{u}$ are $Q$-minima too.

Therefore, recalling the notation in (2.8), we obtain from Lemma 10 of FV08. that

$$
E_{R}(\bar{u})+E_{R}(\underline{u}) \leqslant \operatorname{const} R^{N-1}
$$

as long as $R$ is sufficiently large. 
More precisely, since $\bar{u}$ and $\underline{u}$ are $(N-1) \mathrm{D}$ and $N \leqslant 4$,

$$
\begin{aligned}
& \int_{B_{R}^{N-1}} \frac{1}{p}\left|\nabla \bar{u}\left(x^{\prime}\right)\right|^{p}+W\left(\bar{u}\left(x^{\prime}\right)\right) d x^{\prime} \\
& \quad+\int_{B_{R}^{N-1}} \frac{1}{p}\left|\nabla \underline{u}\left(x^{\prime}\right)\right|^{p}+W\left(\underline{u}\left(x^{\prime}\right)\right) d x^{\prime} \leqslant \operatorname{const} R^{N-2} \leqslant \operatorname{const} R^{2} .
\end{aligned}
$$

This and Proposition 2.11 imply that $\bar{u}$ and $\underline{u}$ are 1D.

Hence, from Theorem 1.1, we conclude that $u$ is 1D.

\section{Proof of Theorem 1.10}

The proof will make use of the results of FV08.

Up to translations, we may suppose that $\xi=e_{N}$.

First of all, by Corollaries 2 and 3 of [FV08, we know that $u_{\epsilon}(x):=u(x / \epsilon)$ converges in $L_{\text {loc }}^{1}$ to the step function $u_{0}:=\chi_{E}-\chi_{\mathbb{R}^{N} \backslash E}$, while $\left\{u_{\epsilon}=\theta\right\}$ approaches $\partial E$ locally uniformly, up to subsequences.

Now fix $K>0$. Then, by (1.14),

$$
\left\{u_{\epsilon}=\theta\right\} \cap\left\{\left|x^{\prime}\right| \leqslant K\right\} \subseteq\left\{\left|x_{N}\right| \leqslant \epsilon \sup _{\left|w^{\prime}\right| \leqslant K} \Phi\left(w^{\prime} / \epsilon, 0\right)\right\} .
$$

Therefore, by (1.15),

$$
\left\{u_{\epsilon}=\theta\right\} \cap\left\{\left|x^{\prime}\right| \leqslant K\right\} \subseteq\left\{\left|x_{N}\right| \leqslant 1 / K\right\}
$$

as long as $\epsilon$ is small enough.

As a consequence,

$$
\partial E \cap\left\{\left|x^{\prime}\right| \leqslant K\right\} \subseteq\left\{\left|x_{N}\right| \leqslant 1 / K\right\}
$$

and so, since $K$ may be taken arbitrarily large,

$$
\partial E \subseteq\left\{x_{N}=0\right\} .
$$

Corollary 7 of FV08 then yields that $u$ is 1D.

\section{REFERENCES}

[AAC01] Giovanni Alberti, Luigi Ambrosio, and Xavier Cabré, On a long-standing conjecture of E. De Giorgi: Symmetry in 3D for general nonlinearities and a local minimality property, Acta Appl. Math. 65 (2001), no. 1-3, 9-33, Special issue dedicated to Antonio Avantaggiati on the occasion of his 70th birthday. MR.1843784 (2002f:35080)

[AC00] Luigi Ambrosio and Xavier Cabré, Entire solutions of semilinear elliptic equations in $\mathbb{R}^{3}$ and a conjecture of De Giorgi, J. Amer. Math. Soc. 13 (2000), no. 4, 725-739 (electronic). MR1775735 (2001g:35064)

[BBG00] Martin T. Barlow, Richard F. Bass, and Changfeng Gui, The Liouville property and a conjecture of De Giorgi, Comm. Pure Appl. Math. 53 (2000), no. 8, 1007-1038. MR:1755949 (2001m:35095)

[BCN97] Henri Berestycki, Luis Caffarelli, and Louis Nirenberg, Further qualitative properties for elliptic equations in unbounded domains, Ann. Scuola Norm. Sup. Pisa Cl. Sci. (4) 25 (1997), no. 1-2, 69-94 (1998), Dedicated to Ennio De Giorgi. MR.1655510 (2000e:35053)

[BHM00] H. Berestycki, F. Hamel, and R. Monneau, One-dimensional symmetry of bounded entire solutions of some elliptic equations, Duke Math. J. 103 (2000), no. 3, 375-396. MR,1763653 (2001j:35069) 
[Bou90] Guy Bouchitté, Singular perturbations of variational problems arising from a twophase transition model, Appl. Math. Optim. 21 (1990), no. 3, 289-314. MR1036589 (91d:49016)

[Dam98] L. Damascelli, Comparison theorems for some quasilinear degenerate elliptic operators and applications to symmetry and monotonicity results, Ann. Inst. H. Poincaré Anal. Non Linéaire 15 (1998), no. 4, 493-516. MR1632933 (99e:35081)

[DG79] Ennio De Giorgi, Convergence problems for functionals and operators, Proceedings of the International Meeting on Recent Methods in Nonlinear Analysis (Rome, 1978) (Bologna), Pitagora, 1979, pp. 131-188. MR533166 (80k:49010)

[DG02] Donatella Danielli and Nicola Garofalo, Properties of entire solutions of non-uniformly elliptic equations arising in geometry and in phase transitions, Calc. Var. Partial Differential Equations 15 (2002), no. 4, 451-491. MR.1942128(2003j:35086)

[DiB83] E. DiBenedetto, $C^{1+\alpha}$ local regularity of weak solutions of degenerate elliptic equations, Nonlinear Anal. 7 (1983), no. 8, 827-850. MR709038 (85d:35037)

[dPKW08] Manuel del Pino, Mike Kowalczyk, and Juncheng Wei, On De Giorgi Conjecture in Dimension $N \geqslant 9$, Preprint (2008), http://eprintweb.org/S/article/math/0806.3141.

[Far99] Alberto Farina, Symmetry for solutions of semilinear elliptic equations in $\mathbb{R}^{N}$ and related conjectures, Ricerche Mat. 48 (1999), no. suppl., 129-154, Papers in memory of Ennio De Giorgi (Italian). MR1765681 (2001h:35056)

[Far01] Monotonicity and one-dimensional symmetry for the solutions of $\Delta u+f(u)=$ 0 in $\mathbb{R}^{N}$ with possibly discontinuous nonlinearity, Adv. Math. Sci. Appl. 11 (2001), no. 2, 811-834. MR.1907468 (2003e:35086)

[Far03] , Rigidity and one-dimensional symmetry for semilinear elliptic equations in the whole of $\mathbb{R}^{N}$ and in half spaces, Adv. Math. Sci. Appl. 13 (2003), no. 1, 65-82. MR2002396 (2004m:35078)

[Far07] , Liouville-type theorems for elliptic problems, Handbook of Differential Equations: Stationary Partial Differential Equations. Vol. IV (M. Chipot, ed.), Elsevier B. V., Amsterdam, 2007, pp. 61-116. MR2569331

[FSV08] Alberto Farina, Berardino Sciunzi, and Enrico Valdinoci, Bernstein and De Giorgi type problems: New results via a geometric approach, Ann. Sc. Norm. Super. Pisa Cl. Sci. (5) 7 (2008), 741-791. MR2483642 (2009j:58020)

[FV08] Alberto Farina and Enrico Valdinoci, Geometry of quasiminimal phase transitions, Calc. Var. Partial Differential Equations 33 (2008), no. 1, 1-35. MR.2413100

[GG98] N. Ghoussoub and C. Gui, On a conjecture of De Giorgi and some related problems, Math. Ann. 311 (1998), no. 3, 481-491. MR1637919 (99j:35049)

[Giu84] Enrico Giusti, Minimal surfaces and functions of bounded variation, Monographs in Mathematics, vol. 80, Birkhäuser Verlag, Basel, 1984. MR775682 (87a:58041)

[HKM06] Juha Heinonen, Tero Kilpeläinen, and Olli Martio, Nonlinear potential theory of degenerate elliptic equations, Dover Publications Inc., Mineola, NY, 2006, Unabridged republication of the 1993 original. MR2305115

[JM04] David Jerison and Régis Monneau, Towards a counter-example to a conjecture of De Giorgi in high dimensions, Ann. Mat. Pura Appl. (4) 183 (2004), no. 4, 439-467. MR.2140525(2005m:35095)

[PSZ99] P. Pucci, J. Serrin, and H. Zou, A strong maximum principle and a compact support principle for singular elliptic inequalities, J. Math. Pures Appl. 78 (1999), no. 8, 769-789. MR 1715341 (2001j:35095)

[PV05] Arshak Petrosyan and Enrico Valdinoci, Density estimates for a degenerate/singular phase-transition model, SIAM J. Math. Anal. 36 (2005), no. 4, 1057-1079 (electronic). MR2139200

[Sav03] Vasile Ovidiu Savin, Phase Transitions: Regularity of Flat Level Sets, Ph.D. thesis, University of Texas at Austin, 2003.

[SV05] Berardino Sciunzi and Enrico Valdinoci, Mean curvature properties for p-Laplace phase transitions, J. Eur. Math. Soc. (JEMS) 7 (2005), no. 3, 319-359. MR2156604 (2006m:35055)

[Tol84] Peter Tolksdorf, Regularity for a more general class of quasilinear elliptic equations, J. Differential Equations 51 (1984), no. 1, 126-150. MR727034 (85g:35047)

[Váz84] J.L. Vázquez, A strong maximum principle for some quasilinear elliptic equations, Appl. Math. Optimization 12 (1984), 191-202 (English). MR768629 (86m:35018) 
[VSS06] Enrico Valdinoci, Berardino Sciunzi, and Vasile Ovidiu Savin, Flat level set regularity of p-Laplace phase transitions, Mem. Amer. Math. Soc. 182 (2006), no. 858, vi+144. MR2228294 (2007a:35050)

Faculté des Sciences, LAMFA - CNRS UMR 6140, Université de Picardie Jules Verne, 33, rue Saint-Leu, 80039 Amiens CEDEX 1, France

E-mail address: alberto.farina@u-picardie.fr

Dipartimento di Matematica, Università di Roma Tor Vergata, Via della ricerca SCIENTIFICA, 1, I-00133 Rome, Italy

E-mail address: enrico@mat.uniroma3.it 